\title{
A complex feature-based representation of vocalizations emerges in the superficial layers of primary auditory cortex
}

Short Title: Vocalization selectivity in the auditory cortex

Authors: Pilar Montes-Lourido ${ }^{1,+, \neq}$, Manaswini Kar ${ }^{1,2, \dagger}$, Stephen V. David ${ }^{3}$, Srivatsun Sadagopan ${ }^{1,2,4,5, *}$

10

Affiliations:

${ }^{1}$ Department of Neurobiology, University of Pittsburgh, Pittsburgh, PA, USA.

${ }^{2}$ Center for Neuroscience, University of Pittsburgh, Pittsburgh, PA, USA.

$15{ }^{3}$ Department of Otolaryngology, Oregon Health and Science University, Portland, OR, USA.

${ }^{4}$ Department of Bioengineering, University of Pittsburgh, Pittsburgh, PA, USA.

${ }^{5}$ Center for the Neural Basis of Cognition, University of Pittsburgh, Pittsburgh, PA, USA.

${ }^{\dagger}$ Equal contribution

${ }^{\ddagger}$ Current address: Department of Transfer and Innovation, USC University Hospital Complex

20 (CHUS), University of Santiago de Compostela, Spain.

*Correspondence to:

Srivatsun Sadagopan

University of Pittsburgh/Neurobiology

$35015^{\text {th }}$ Ave,

25 Biomedical Science Tower - 3, Room 10021

Pittsburgh, PA 15261

Phone: 412-624-8920

Email: vatsun@pitt.edu 


\section{Abstract}

Early in auditory processing, neural responses faithfully reflect acoustic input. At higher stages of auditory processing, however, neurons become selective for particular call types, eventually leading to specialized regions of cortex that preferentially process calls at the highest auditory processing stages. We previously proposed that an intermediate step in how non-selective responses are transformed into call-selective responses is the detection of informative call features. But how neural selectivity for informative call features emerges from non-selective inputs, whether feature selectivity gradually emerges over the processing hierarchy, and how stimulus information is represented in non-selective and feature-selective populations remain open questions. In this study, using unanesthetized guinea pigs, a highly vocal and social rodent, as an animal model, we characterized the neural representation of calls in three auditory processing stages - the thalamus (VMGB), and thalamorecipient (L4) and superficial layers (L2/3) of primary auditory cortex (A1). We found that neurons in VMGB and A1 L4 did not exhibit callselective responses and responded throughout the call durations. However, A1 L2/3 neurons

45 showed high call-selectivity with about a third of neurons responding to only one or two call types. These A1 L2/3 neurons only responded to restricted portions of calls suggesting that they were highly selective for call features. Receptive fields of these A1 L2/3 neurons showed complex spectrotemporal structures that could underlie their high call feature selectivity. Information theoretic analysis revealed that in A1 L4 stimulus information was distributed over the population and was spread out over the call durations. In contrast, in A1 L2/3, individual neurons showed brief bursts of high stimulus-specific information, and conveyed high levels of information per spike. These data demonstrate that a transformation in the neural representation of calls occurs between $A 1 L 4$ and $A 1 L 2 / 3$, leading to the emergence of a feature-based representation of calls in $A 1 \mathrm{~L} 2 / 3$. Our data thus suggest that observed cortical specializations for call processing emerge in $\mathrm{A} 1$, and set the stage for further mechanistic studies.

\section{Introduction}

How behaviorally critical sounds, such as conspecific vocalizations (calls), are represented in the activity of neural populations at various stages of the auditory processing hierarchy is a central

60 question in auditory neuroscience. Early representations of sounds, such as in the auditory nerve, have been proposed to be optimized for the efficient and faithful representation of sounds in general [1, 2]. Consequently, at lower auditory processing stations, vocalizations are not represented any differently than other sounds ([3, 4]; but see [5]). At the other extreme, behaviorally-relevant stimuli such as vocalizations are over-represented at the highest cortical 
65 processing stages [6-9]. In macaques and marmosets, neurons in the highest stages of the auditory processing hierarchy show strong selectivity for call category and even caller identity [10-12]. How the neural representation of calls is transformed from a nonspecific format in early processing stages to a call-selective format at higher processing stages remains unclear. Because auditory receptive fields increase in complexity as one ascends the auditory processing hierarchy $[13,14]$, the conventional hypothesis is that call selectivity is gradually refined across auditory processing stages. However, there is little systematic evidence supporting a gradual refinement in call selectivity. While many studies have investigated call representations in subcortical and cortical stages [6, 7, 15-27], these have not systematically explored the mechanisms of how call representations could be transformed from one stage to the next, or how

75 this impacts information representation at different processing stages. A clear understanding of where critical transformations occur is an essential first step in designing experiments to probe neural mechanisms underlying these transformations, and to target these experiments to the appropriate processing stage in the auditory hierarchy. In this study, we recorded neural responses to an extensive set of call stimuli across multiple auditory processing stages to test

80 whether the emergence of call selectivity is gradual, and to characterize the nature and informativeness of call representations at these processing stages.

The first question to address is what it means for a neuron to be call selective. In many mammalian species, calls are not produced stereotypically from trial to trial; rather, calls are

85 instantiations of an underlying noisy production process. Thus, there is considerable variability in the production of calls belonging to a given call category both across trials and across individuals $[28,29]$. Furthermore, different call categories may have highly overlapping spectral content. To be call category selective, a neuron has to be selective for more than purely spectral cues, and has to generalize across production variability. In previous theoretical work, we showed that in

90 order to construct high level call category-selective neural responses, it is first necessary to have an intermediate representation where neurons detect informative call features [29]. Informative call features are spectrotemporal fragments of calls that are most likely to be found across exemplars of a given category (despite production variability), and typically span about an octave in frequency and about a hundred milliseconds in time. Thus, if one of the objectives of cortical

95 processing is call categorization, our model would predict the existence of diverse neurons, each tuned for model-predicted informative features. Consistent with this prediction, limited experimental data suggested that call feature-selective neurons could be found in primary auditory cortex (A1) of marmosets and guinea pigs (GPs) [29]. But the question remains whether 
feature-selectivity is gradually constructed over the ascending auditory pathway, or if it emerges de-novo at some processing stage.

At lower processing stations of the auditory pathway in GPs and non-human primates, there is little evidence for the existence of call feature-selective neurons [15, 16, 22]. Rather, neurons appear to respond to call types in a manner largely explained by frequency tuning [15, 105 16, 22]. In GPs, single neurons in the inferior colliculus (IC) are not selective for particular call types or call features [16]. In primates and GPs, even at the level of A1, many previous studies have not reported strong selectivity for particular call types or features, or preference for natural over reversed calls $([17,20,21,30]$; but see below). It is only at the level of secondary cortex that clear call-selective responses have been reported, both in primates (in anterolateral belt, AL; [8, 110 9]), and in GPs (Area S and the ventral-rostral belt, VRB [6]). However, gaps in understanding remain because of some technical limitations of these studies, including the use of anesthesia, limited stimulus sets, multi-unit recordings, or not comparing across processing stages, specifically across cortical laminae. Thus, these studies do not give rise to a clear picture of where and how a call feature-specific representation first emerges.

A few studies have provided hints that A1 could be a locus of important transformations to the neural representation of calls. In A1 of awake squirrel monkeys, one study reported that about a third of neurons responded to call stimuli that showed similarities in their frequency-time characteristics [23]. In marmoset A1, about a third of A1 neurons at shallower recording depths 120 showed highly non-linear receptive fields that could in turn underlie call feature selectivity [31]. It has been proposed that because A1 neurons cannot phase-lock to fast envelope fluctuations, sparse spiking in A1 could provide temporal markers that reflect subcortical spectrotemporal integration [32]. But these studies did not specify whether recordings were from the input or output layers of $A 1$. In humans, a recent study using ultra high-field fMRI with laminar resolution reported

125 that whereas BOLD activity in granular and infragranular layers could be explained using simple frequency content based models, activity in supragranular layers could be explained better using more complex models incorporating spectral and temporal modulations [33]. This supragranular activity resembled activity in secondary auditory cortical areas, suggesting that a transformation between thalamorecipient ( $A 1 L 4)$ and superficial $(A 1 L 2 / 3)$ layers of $A 1$ might give rise to more 130 specialized processing. Thus, a careful investigation of the thalamus and across identified cortical laminae of $\mathrm{A} 1$ is necessary to understand how the cortex might transform sound representations, particularly with respect to behaviorally critical sounds such as calls. 
In this study, we begin to address how early nonspecific and spectral content based representations are transformed into higher feature-based representations. We recorded neural

135 activity from unanesthetized GPs passively listening to an extensive range of conspecific calls $[6,34,35]$, and acquired single-unit responses from the thalamus (vMGB), thalamorecipient (A1 L4), and superficial (A1 L2/3) layers of A1. We found that neurons in VMGB and A1 L4 responded to most call categories and throughout the call durations. In contrast, a third of A1 L2/3 neurons responded sparsely and selectively to one or two call categories, and only in specific time bins

140 within a call. These A1 L2/3 neurons showed highly complex receptive fields that could underlie this call feature selectivity. Information theoretic analyses revealed that while average mutual information (MI) was high in A1 L4, Ml was about evenly distributed over the population of neurons and across multiple stimuli, and sustained over the stimulus duration. In contrast, individual A1 L2/3 neurons were highly informative about few stimuli, and conveyed high levels of information

145 per spike in only a handful of time bins. These results argue against a gradual emergence of call feature selectivity, and suggest that a significant transformation in the neural representation of calls occurs between $A 1 L 4$ and $A 1 L 2 / 3$, leading to the emergence of a feature-based representation of calls in $\mathrm{A} 1 \mathrm{~L} 2 / 3$.

\section{Results}

We recorded the activity of single neurons located in the VMGB, A1 L4, and A1 L2/3 of unanesthetized, head-fixed, passively-listening GPs (Fig. 1A, top). We first implanted a headpost and recording chambers onto the skull of the animals using aseptic surgical technique. We then performed small craniotomies $(\sim 1.0 \mathrm{~mm}$ diameter) to access the underlying tissue (Fig. 1A, 155 bottom). Single-unit activity was recorded using high-impedance tungsten electrodes and first sorted online using a template-match algorithm, and later refined offline. Over a few weeks, we sequentially recorded from a number of such craniotomies and constructed tonotopic maps (Fig. 1C). The location of $A 1$ was confirmed using the direction of the tonotopic gradient and tonotopic reversals. Note that in GPs, the $\mathrm{A} 1$ gradient is similar to primates, and runs from low frequencies

160 rostrally to high frequencies caudally [6, 36, 37]. On each track, we also acquired local field potential (LFP) responses to tones at evenly-spaced depths, from which we calculated the current source density (CSD) profile of the track (Fig. 1B). The thalamorecipient layers (referred to here as A1 L4) were identified based on the presence of a short-latency current sink and LFP polarity reversal [38]. We distinguished between regular-spiking (RS) and fast-spiking (FS) neurons in our

165 recordings using spike width and peak-to-trough amplitude ratio (Fig. 1D). About 20\% of our recordings were from FS neurons, but call responses were tested in only half these neurons. Only 
RS neurons are reported in this study. Spontaneous rates of A1 L2/3 neurons (Fig. 1E; median: $1.51 \mathrm{spk} / \mathrm{s}$ ) were not significantly different from A1 L4 neurons (median: $2.31 \mathrm{spk} / \mathrm{s}$ ), but were significantly lower compared to vMGB neurons (median: $3.67 \mathrm{spk} / \mathrm{s}$; Kruskal-Wallis test $\mathrm{p}=0.008$; post-hoc Dunn-Sidak tests vMGB vs. A1 L4: $p=0.1112, A 1$ L2/3 vs. vMGB: $p=0.005, A 1$ L2/3 vs. A1 L4: $p=0.085)$. We sampled over a broad range of neural best frequencies that overlapped with the call frequency range (Fig. 1F). Pure tone tuning bandwidths of tone-responsive neurons at all processing stages showed a dependence on best frequency (Fig. 1G; ANOCOVA with best frequency as co-variate, $p=0.0071$ ), and after controlling for this frequency dependence, the

175 bandwidths of VMGB neurons were significantly higher than A1 L2/3 neurons (ANOCOVA constrained to same slopes; intercept effect $p=0.0017$; post-hoc Tukey's HSD vMGB vs. A1 L4: $p=0.053, A 1 L 2 / 3$ vs. vMGB: $p=0.0012)$. A1 L4 and A1 L2/3 bandwidths were not significantly different $(p=0.554)$. Following basic characterization, we presented a range of GP calls (8 categories, 2 or more exemplars of each category; Fig. 2). Note that our vocalization set did not 180 have acoustic power in the $4-6 \mathrm{kHz}$ range, which may explain the relative paucity of callresponsive neurons we encountered in that range, particularly in cortical recordings. All call categories were about evenly represented in neural responses across the processing stages (Fig. $1 \mathrm{H})$. The only statistically significant deviations we observed was a small over-representation of 'Other' calls and a small under-representation of 'Purr' calls in A1 L2/3 ( $p=0.014$ for both, twosided permutation test with FDR correction for 24 comparisons). All further analyses are based only on call-responsive neurons from the vMGB $(n=33), A 1 L 4(n=67)$, and A1 L2/3 $(n=45)$.

(continued on next page) 


\section{Figure 1}
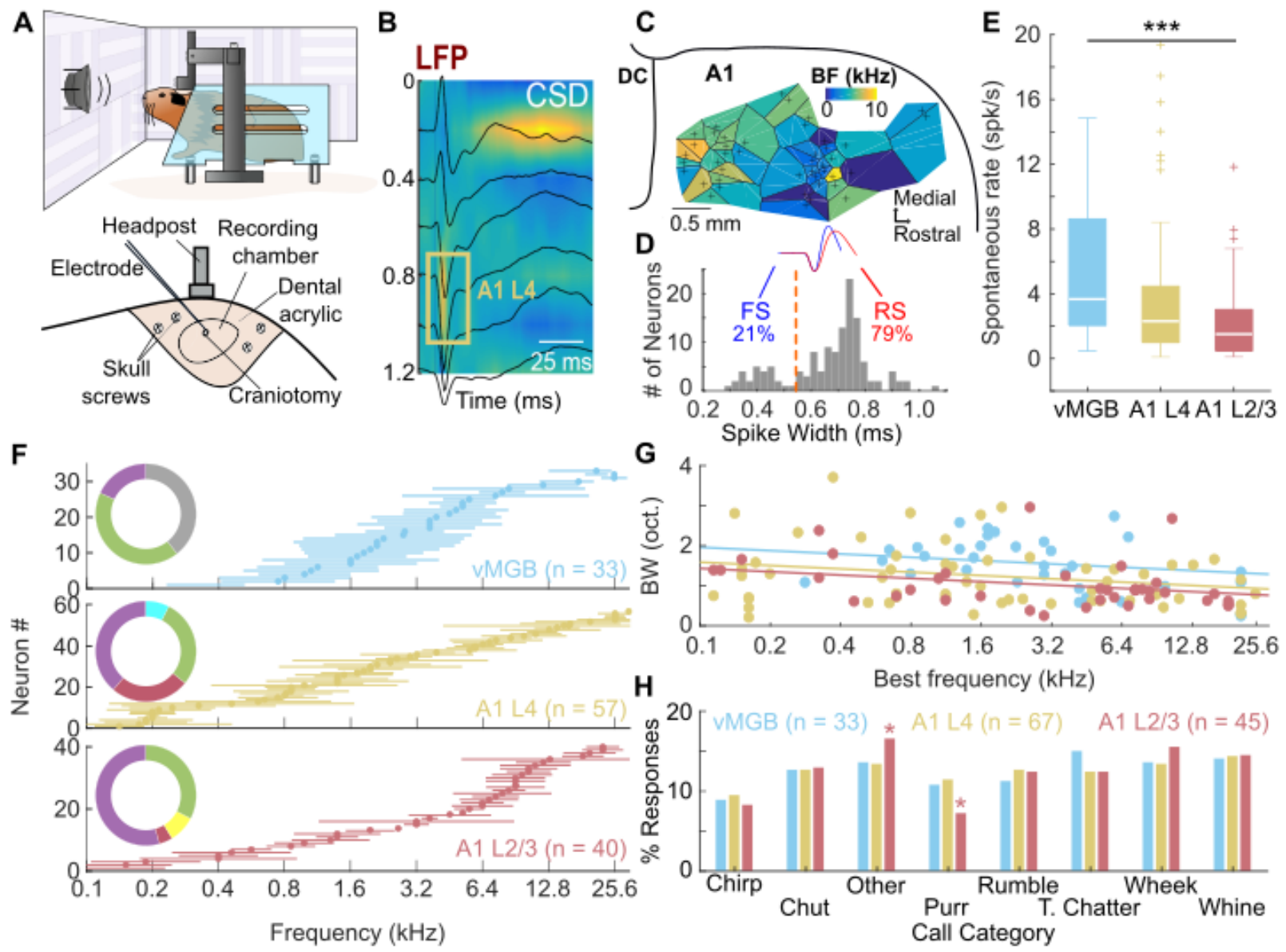

Figure 1: Single-unit recordings from unanesthetized, head-fixed guinea pigs.

(A) Recording setup (top) and details of cranial implant (bottom). (B) Average LFP traces (black lines) and CSD (colormap; warm colors correspond to sinks) of an example electrode track in A1. Yellow box outlines estimated A1 L4 location. (C) Example Voronoi map showing tonotopy of auditory cortex in one GP. Colormap corresponds to best frequency. (D) Histogram of spike widths of sorted single units. Dashed orange line is the threshold used to separate FS (blue) from RS (red) units. (E) Distribution of spontaneous rates in VMGB (blue), A1 L4 (yellow) and A1 L2/3 (red).

$215{ }^{* * *}: p<0.005$, Kruskal-Wallis test (Dunn-Sidak post-hoc test). (F) Best frequencies (discs) and bandwidths (lines) of tone-responsive neurons recorded from vMGB, A1 L4 and A1 L2/3 (colors as earlier). Insets show distribution of units across subjects, colors correspond to individual subjects. (G) Tone tuning bandwidth plotted as a function of best frequency across all three auditory stages tested. Dots correspond to individual neurons and lines correspond to linear fits

220 constrained to have the same slope. (H) Fraction of call-responsive neurons in vMGB, A1 L4, and A1 L2/3 that respond to each call category ( ${ }^{*}: p<0.05$, two-sided permutation test with FDR correction). 


\section{Figure 2}

A

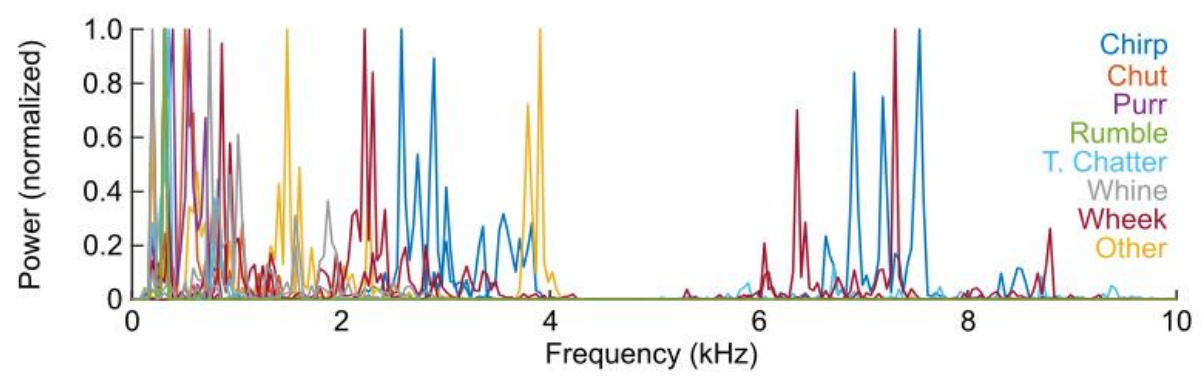

B
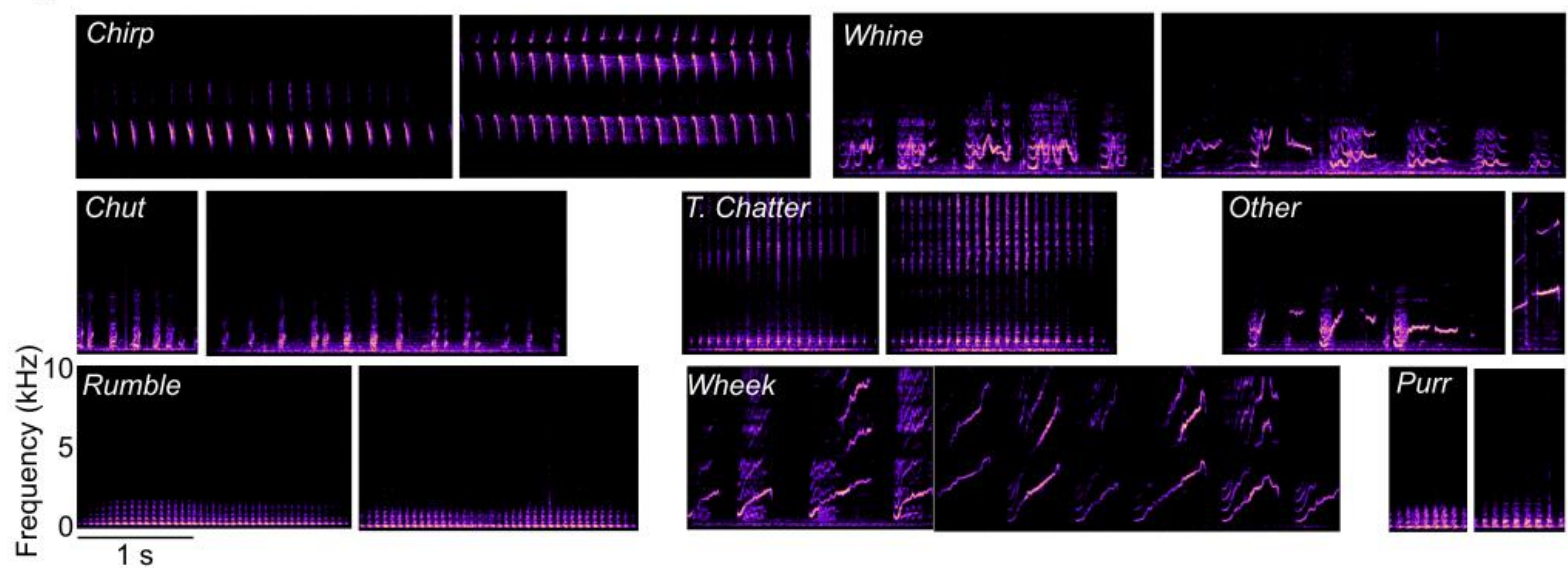

230 Figure 2: Spectra and spectrograms of guinea pig calls.

(A) Normalized power spectra of the guinea pig calls used in this study. Colors correspond to different call categories. (B) Spectrograms of the guinea pig calls used in this study (8 categories, 2 calls per category).

\section{Call selectivity emerges in superficial cortical layers}

Call selectivity could emerge through a gradual sharpening of tuning along successive stages of the ascending auditory pathway, or could sharply emerge at some processing stage. To distinguish between these models, we quantified the call selectivity of neural populations in vMGB, A1 L4 and A1 L2/3. Figure 3 shows representative examples of neural responses to calls

240 in vMGB (Fig. 3A), A1 L4 (Fig. 3B), and A1 L2/3 (Fig. 3C). Neurons in vMGB and A1 L4 typically responded to many call categories, with responses sustained throughout the call, or occurring at multiple times over the duration of a call. In contrast, neurons in A1 L2/3 responded to very few calls, and only for short durations within each call.

Conventionally, response rates and response significance are calculated over a fixed response window, typically encompassing the entire stimulus duration. For a first-pass analysis, we defined selectivity as the number of call categories that, compared to spontaneous rate, 
evoked a significant response over the entire call duration (1 - highly selective, 8 - no selectivity). The median selectivity of the A1 L2/3 population was 3 call categories, whereas the medians for

250 the A1 L4 and VMGB populations were 6 call categories ( $p=3.5 \times 10^{-6}$; Kruskal-Wallis test). While this approach accurately estimated response properties when response rates were high and sustained, it sometimes failed to capture feature-selective responses that were restricted to only some time bins of the stimulus, such as those we observed in $A 1 \mathrm{~L} 2 / 3$. To overcome this limitation, we used an automated procedure to estimate significant response windows for each stimulus

255 (orange boxes in Fig. 3; see Methods). If at least one response window was detected for any exemplar belonging to a call category, we conservatively counted the neuron as being responsive to that category.

Over the population of recorded neurons, while VMGB and A1 L4 neurons showed 260 significant responses to most of the categories tested (Fig. 4A left and center, median of 7 categories for both VMGB and A1 L4), nearly a third of $A 1 L 2 / 3$ neurons responded to only one or two call categories (Fig. 4A right; median =5). Distributions of call selectivity were not significantly different between the VMGB and A1 L4 populations (medians = 7). In contrast, A1 L2/3 neurons responded to significantly fewer categories of calls $(p=2.8 \times 10-5$, Kruskal-Wallis test; post-hoc

265 Dunn-Sidak corrected $p$-values are: vMGB vs. A1 L4: $p=0.90 ; A 1$ L2/3 vs. vMGB: $p=2.5 \times 10^{-4}$; $A 1 L 2 / 3$ vs. $A 1 L 4: p=1.9 \times 10^{-4}$ ). The temporal characteristics of the response and response duration are shown in Fig. 4B, where we plot the joint distribution of the number of response windows found per call and the fractional length of call stimuli spanned by response windows in vMGB, A1 L4, and A1 L2/3. While most vMGB and A1 L4 neurons typically exhibited two or more

270 response windows per call that spanned a larger fraction of call length, many A1 L2/3 neurons usually exhibited only one response window per call with response windows spanning a smaller fraction of call length. The temporal response characteristics of vMGB and A1 L4 were therefore not significantly different ( $p=0.48,2-D$ K-S [39] test with Bonferroni correction), whereas A1 L2/3 responses were significantly different (A1 L2/3 vs. vMGB: $p=0.0008, A 1$ L2/3 vs. A1 L4: $p=$

$2750.0023 ; 2-D$ K-S test with Bonferroni correction). Thus, at the culmination of subcortical processing, vMGB responses are not call selective, and in fact mirror earlier studies showing a lack of call selectivity in GP IC [16]. Even at the first cortical processing stage (A1 L4), no transformation to the representation of calls seems to have occurred. However, our data demonstrate that a significant transformation to call representation occurs in many superficial 280 cortical neurons (A1 L2/3). These data strongly support the de-novo emergence of call featureselective responses in the superficial layers of primary auditory cortex. 
Figure 3
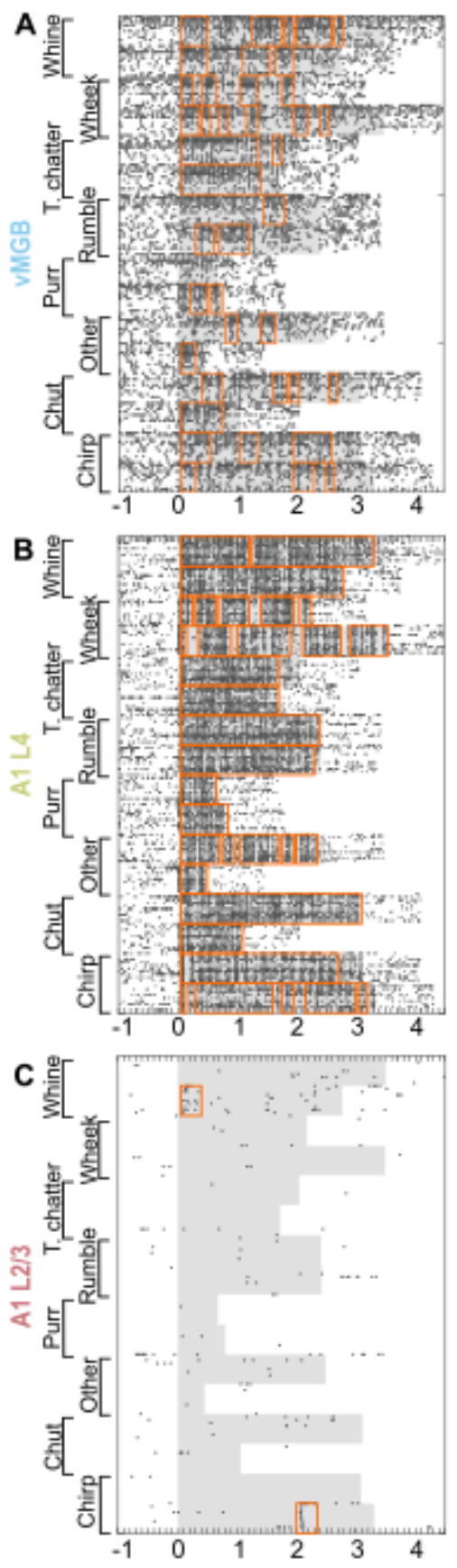
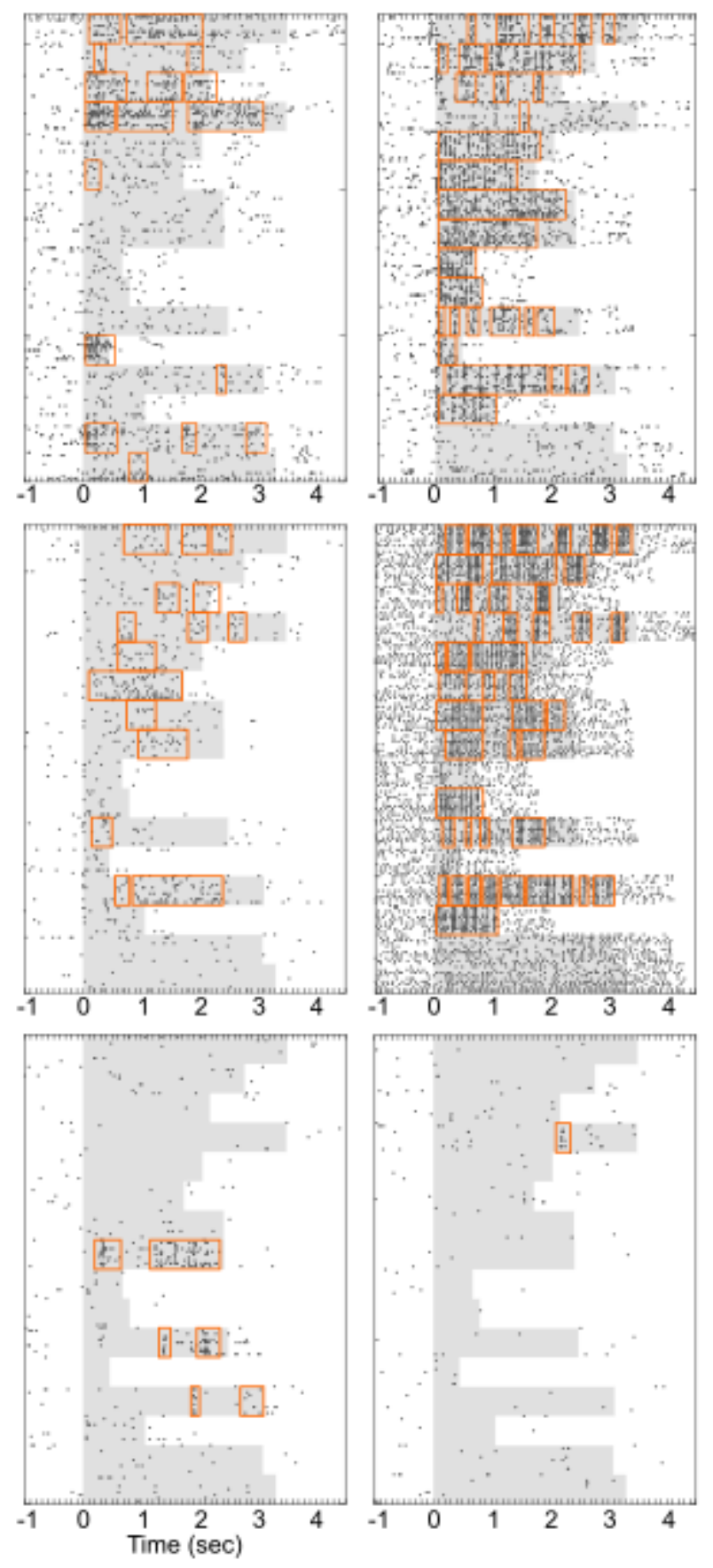

Figure 3: Detection of response windows.

Spike rasters of three call-responsive neurons from (A) VMGB, (B) A1 L4, and (C) A1 L2/3 are 285 plotted. Gray shading indicates stimulus duration, and black dots correspond to spike times. Orange boxes correspond to response windows detected using our algorithm. 


\section{Figure 4}

A
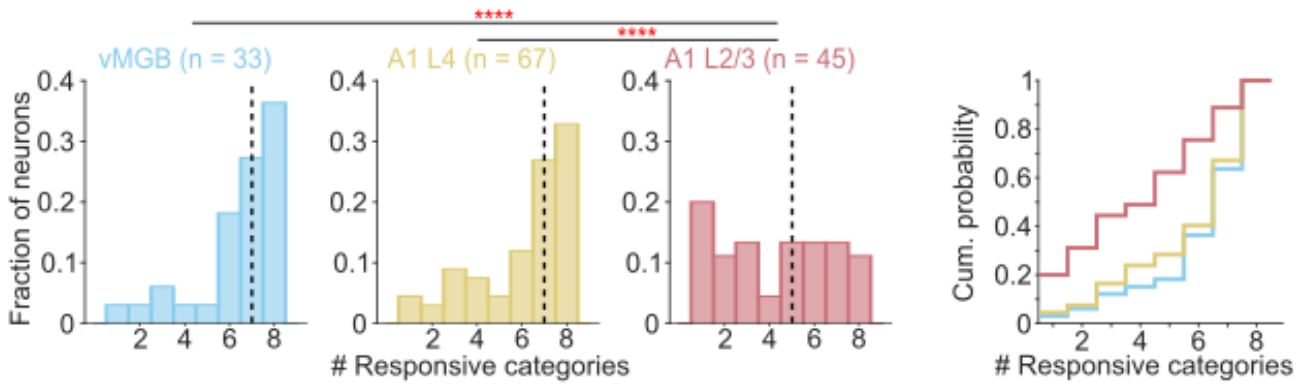

B
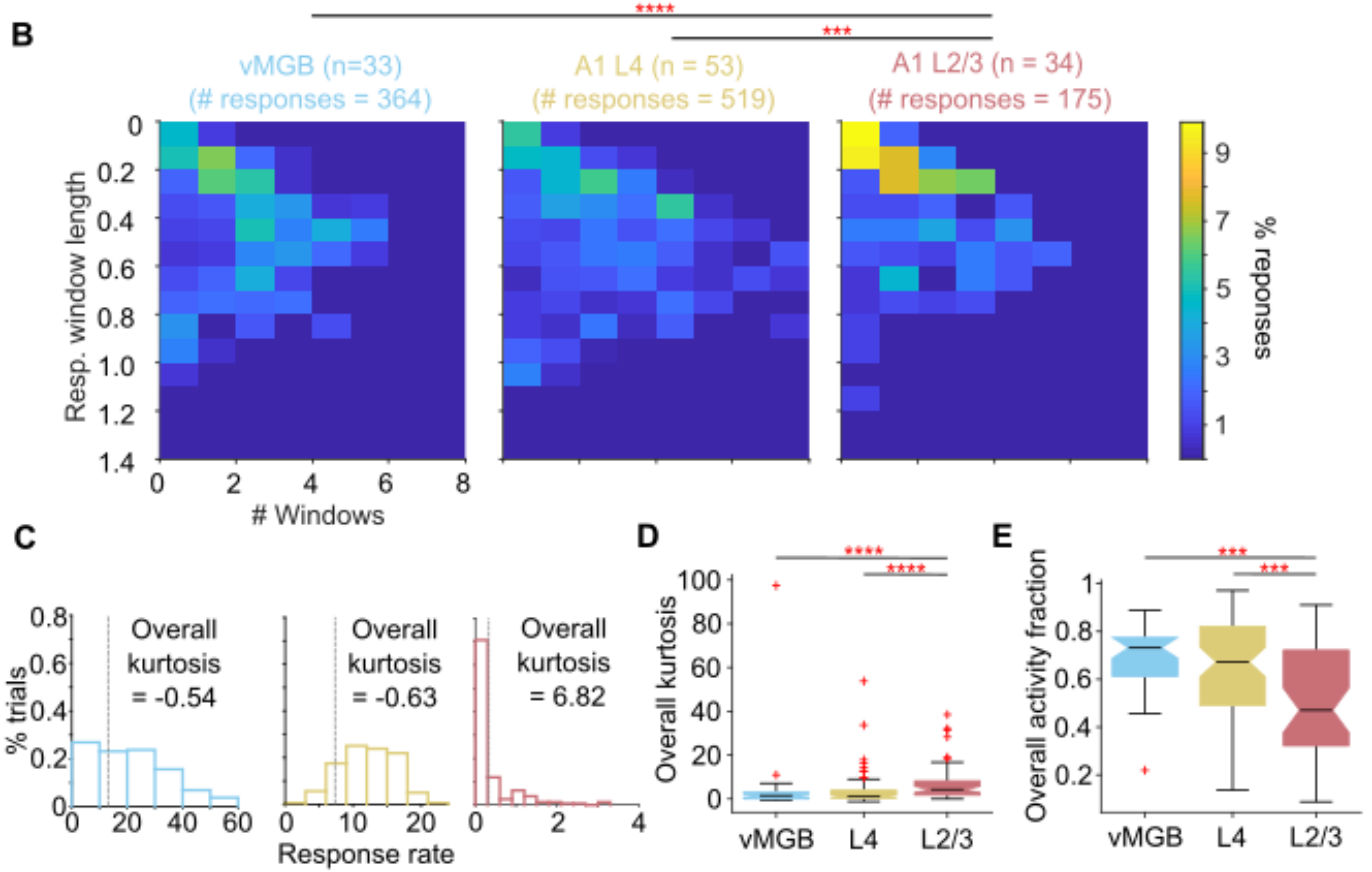

(spikes/s)

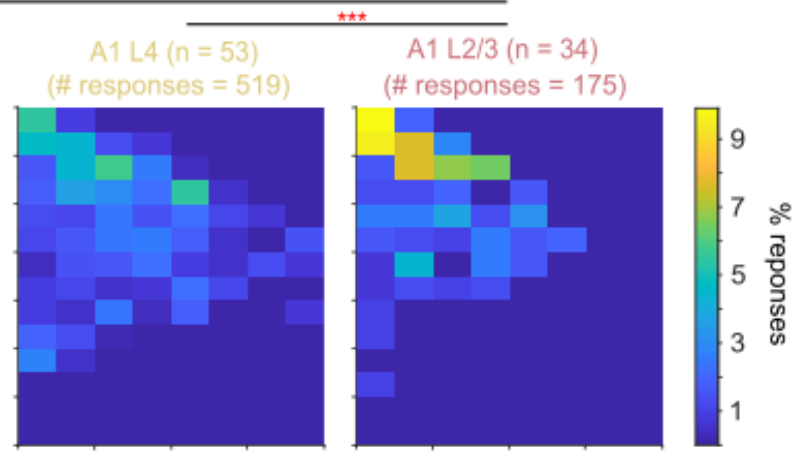

290

Figure 4: Neural selectivity for call features emerges in A1 L2/3.

(A) Distributions of call selectivity in VMGB (blue), A1 L4 (yellow), and A1 L2/3 (red). Black dashed lines are medians. Comparison of cumulative distributions is shown on the right. (B) Joint distributions of the number of response windows and the fractional length of the call stimuli spanned by all windows exhibited by neurons at the different processing stages. vMGB and A1 L4 neurons tended to exhibit either multiple short windows or a single long window that spanned a large portion of the stimuli. In contrast, A1 L2/3 neurons exhibited one or two short response windows. (C) Distributions of trial-wise response rates in an example vMGB (blue; same neuron as in Fig. 3A, left), A1 L4 (yellow; same neuron as in Fig. 3B, left) and A1 L2/3 (red; same neuron as in Fig. 3C, left) neuron. Kurtosis values calculated over the entire call length are shown. Gray dashed line corresponds to spontaneous rate. (D) Distributions of sparseness (kurtosis) across auditory processing stages. A1 L2/3 responses were significantly sparser than A1 L4 and vMGB responses. (E) Same as (D) but with activity fraction used as a metric of response sparseness. For all panels except B, Kruskal-Wallis tests with posthoc Dunn-Sidak tests were used for 305 statistical comparisons. For B, a two-dimensional KS test with Bonferroni correction was used. Asterisks correspond to: ${ }^{*}: p<0.05,{ }^{* *}: p<0.01,{ }^{* * *}: p<0.005,{ }^{* * *}: p<0.001$ (exact $p$-values in main text). 
To evaluate whether neurons specifically responded to only parts of some calls or if neural responses were more evenly distributed across calls using metrics independent of stimulus identity and response window detection parameters, we characterized response sparsity. We defined sparseness as 1) the reduced kurtosis of the trial-wise firing rate distribution, and 2) the activity fraction ([40,41]; see Equation 1) of the trial-wise responses. For neurons that responded to most trials about evenly, such as the A1 L4 neuron in Fig. 3B (left), the firing rate distribution was approximately normal, resulting in low kurtosis values (Fig. 4C, center). In contrast, for 315 neurons that responded strongly only on some trials, and were unresponsive for most trials, such as the $\mathrm{A} 1 \mathrm{~L} 2 / 3$ neuron in Fig. 3C (left), the firing rate distribution showed high kurtosis (Fig. 4C, right). Over the population, for both sparsity metrics (kurtosis, Fig. 4D; and activity fraction, Fig. $4 \mathrm{E}$ ), we found that $\mathrm{VMGB}$ and $\mathrm{A} 1 \mathrm{~L} 4$ responses were not sparse and not significantly different from each other. Consistent with earlier analyses, compared to both vMGB and A1 L4, A1 L2/3

320 responses were highly sparse and sparsity distributions were significantly different (Kurtosis: $p=$ $3.2 \times 10^{-5}$, Kruskal-Wallis test; Dunn-Sidak posthoc test $p$-values are: vMGB vs. A1 L4: $p=0.99$, A1 L2/3 vs. vMGB: $p=5.5 \times 10^{-4}, A 1 \mathrm{~L} 2 / 3$ vs. A1 L4: $p=1.2 \times 10^{-4}$. Activity fraction: $p=5.2 \times 10^{-}$ ${ }^{4}$, Kruskal-Wallis test; Dunn-Sidak posthoc test $p$-values are: vMGB vs. A1 L4: $p=0.79, A 1$ L2/3 vs. vMGB: $p=0.001, A 1$ L2/3 vs. A1 L4: $p=0.004$ ).

These observed differences in A1 L2/3 selectivity and sparsity could not simply be attributed to differences in frequency tuning. As mentioned above, pure tone tuning bandwidths of tone-responsive neurons in A1 L2/3 were not significantly different from A1 L4 neurons (Fig. $1 G)$. High call selectivity in A1 L2/3 could also arise if only a few call types are over-represented 330 in this processing stage. This was not the case in our data - as described earlier, neural preference for call type was about evenly distributed across all tested call types across the processing stages. These controls thus suggest that the emergence of call or feature selectivity in $\mathrm{A} 1 \mathrm{~L} 2 / 3$ is the consequence of cortical computations that result in a meaningful transformation of information representation between processing stages.

Because responses were evoked for more call categories and for larger fractional lengths of the calls in VMGB and A1 L4, and given the overlapping spectral content of call categories that is largely maintained over the call durations (Fig. 2), we hypothesized that VMGB and A1 L4 neurons were likely driven by the spectral content of calls, responding when call spectral energy 340 overlapped with the neurons' tone receptive fields. In contrast, despite this overlap of spectral energy across call types, many A1 L2/3 neurons responded to few call types and only in narrow 
windows, suggesting that they were likely driven by specific spectrotemporal features that occur during calls, consistent with our earlier theoretical model [29]. We tested these hypotheses by estimating the spectrotemporal receptive fields (STRFs) that best explained neural responses

345 across the processing stages.

\section{Complex spectrotemporal features drive call-selective responses}

To determine the call features driving neural responses, we used the Neural Encoding Model System (NEMS [42,43]; https://github.com/LBHB/NEMS) to fit linear-nonlinear (LN) models to

350 neural responses to calls. The input to these models was the concatenated cochleagram of all call stimuli (6 oct. frequency range with 5 steps/oct; 20 ms time bins; $\sim 35 \mathrm{~s}$ total; Fig. 5B), constructed using a fast approximation algorithm based on a weighted log-spaced spectrogram and three rate-level transformations corresponding to three categories of auditory nerve fibers ([44]; https://github.com/monzilur/cochlear_models). A recent study demonstrated that such an

355 input representation adequately captures the auditory input to cortex for the purposes of receptive field estimation [44]. The objective of the encoding model was to estimate a set of linear weights (the STRF of the neuron), which when convolved with the input cochleagram and then transformed through a point nonlinearity, would yield a predicted peristimulus time histogram (PSTH; Fig. 5A; see Methods for details). The correlation coefficient between predicted PSTHs of

360 validation segments of neural responses (labeled $r$ in figures; see Methods) and actual response PSTHs was used as the performance metric. For display and measuring STRF sparsity, we used significance-masked average STRFs (see Methods).

Examples of STRF estimates and comparisons of predicted responses to observed 365 responses are shown for neurons with a range of call selectivities from different subjects in A1 L4 and $A 1$ L2/3 in figures 5 and 6 . For many A1 L4 neurons (Fig. 5), STRF estimates that best captured the response showed a clear tuning for specific frequencies, and significant weights were restricted to a narrow range of frequencies and few time bins. While a few call-selective A1 L4 responses could not be directly explained by call energy overlapping with an excitatory 370 receptive field subunit (for example, Fig. 5C, D), responses of most A1 L4 neurons to calls occurred when call energy was present within the excitatory subunits of the receptive fields (horizontal blue lines in Fig. 5E - H). In contrast, STRFs of A1 L2/3 neurons estimated using the same procedure were often more complex (Fig. 6). We observed STRFs with preferences for repetitive features (Fig. 6A), harmonically-related features (Fig. 6G), and frequency-modulated 375 features (Fig. 6K). Compared to A1 L4 estimates, significant A1 L2/3 weights spanned a greater 
range of frequencies and time bins. When we overlaid different stimulus segments on the A1 L2/3 STRFs, we observed that responses did not occur when only stimulus spectral energy matched STRF excitatory subunits (red boxes labeled '3', '4', and '5' in Fig. 6). Rather, responses were elicited when complex stimulus features matched multiple STRF subunits (green boxes labeled ' 1 ' and ' 2 ' in Fig. 6).

\section{Figure 5}
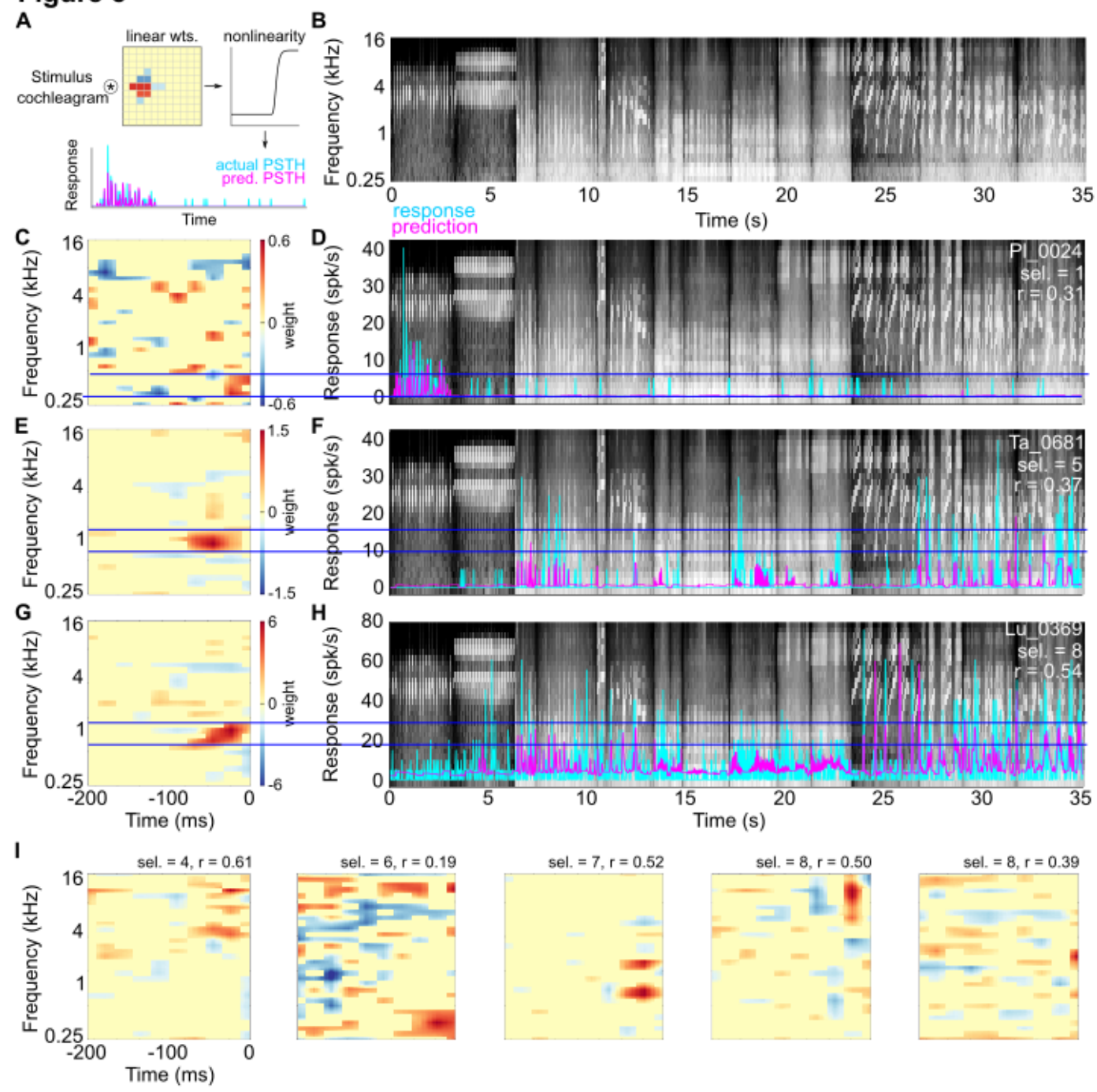

Figure 5: STRF estimates of example A1 L4 neurons.

(A) Schematic of the linear-nonlinear model architecture used to estimate STRFs. (B) Stimulus cochleagram of 16 call stimuli (8 categories) used as the input to the model. (C, E, G) Mean STRF estimates of three A1 L4 neurons with a range of selectivity values. (D, F, H) Comparison of predicted PSTHs (magenta) and observed responses (cyan) of these three neurons. Horizontal blue lines denote the extent of the frequency tuning of the STRFs. (I) Additional examples of A1 L4 STRF estimates (sel. = call selectivity, $r=$ correlation between predicted and actual responses derived from the validation data set). 


\section{Figure 6}
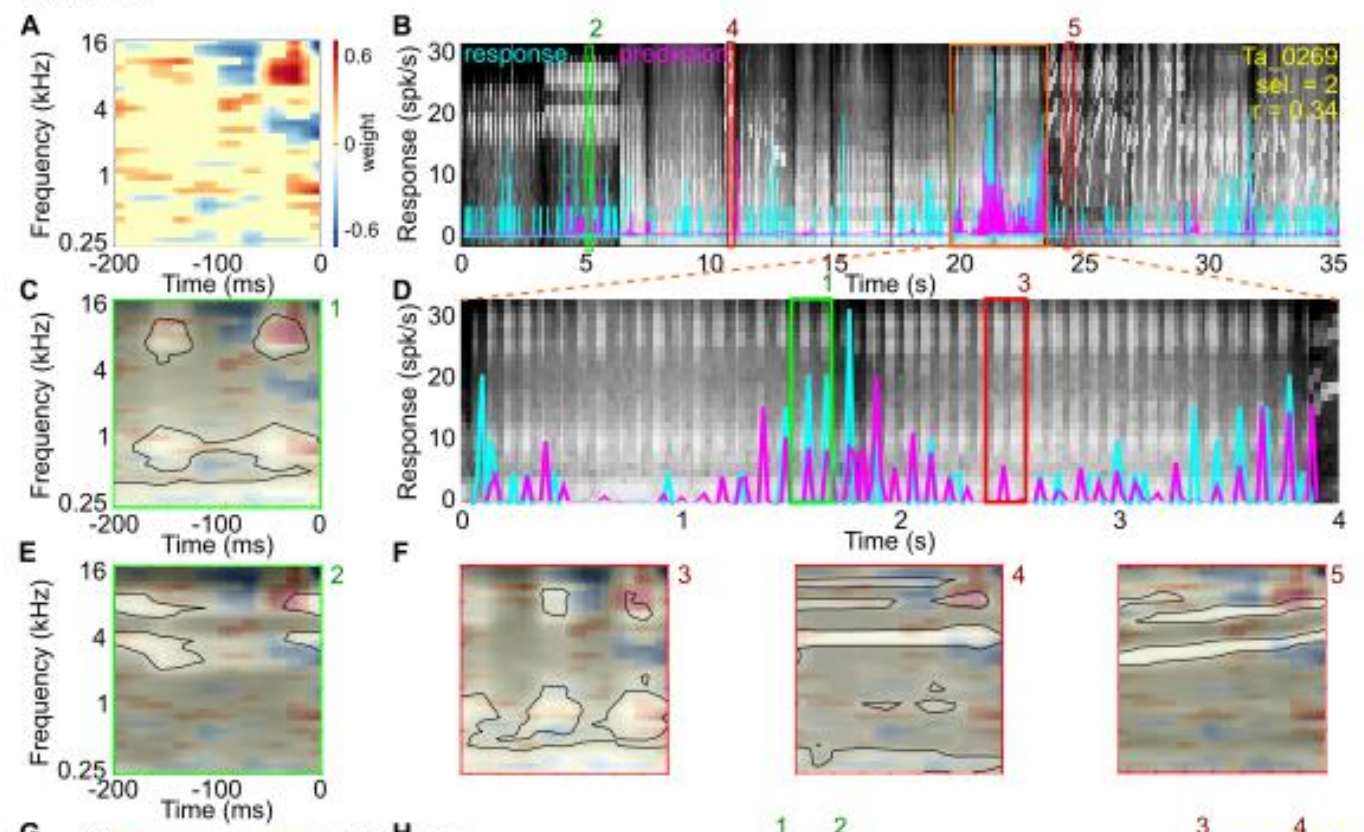

$\mathbf{F}$
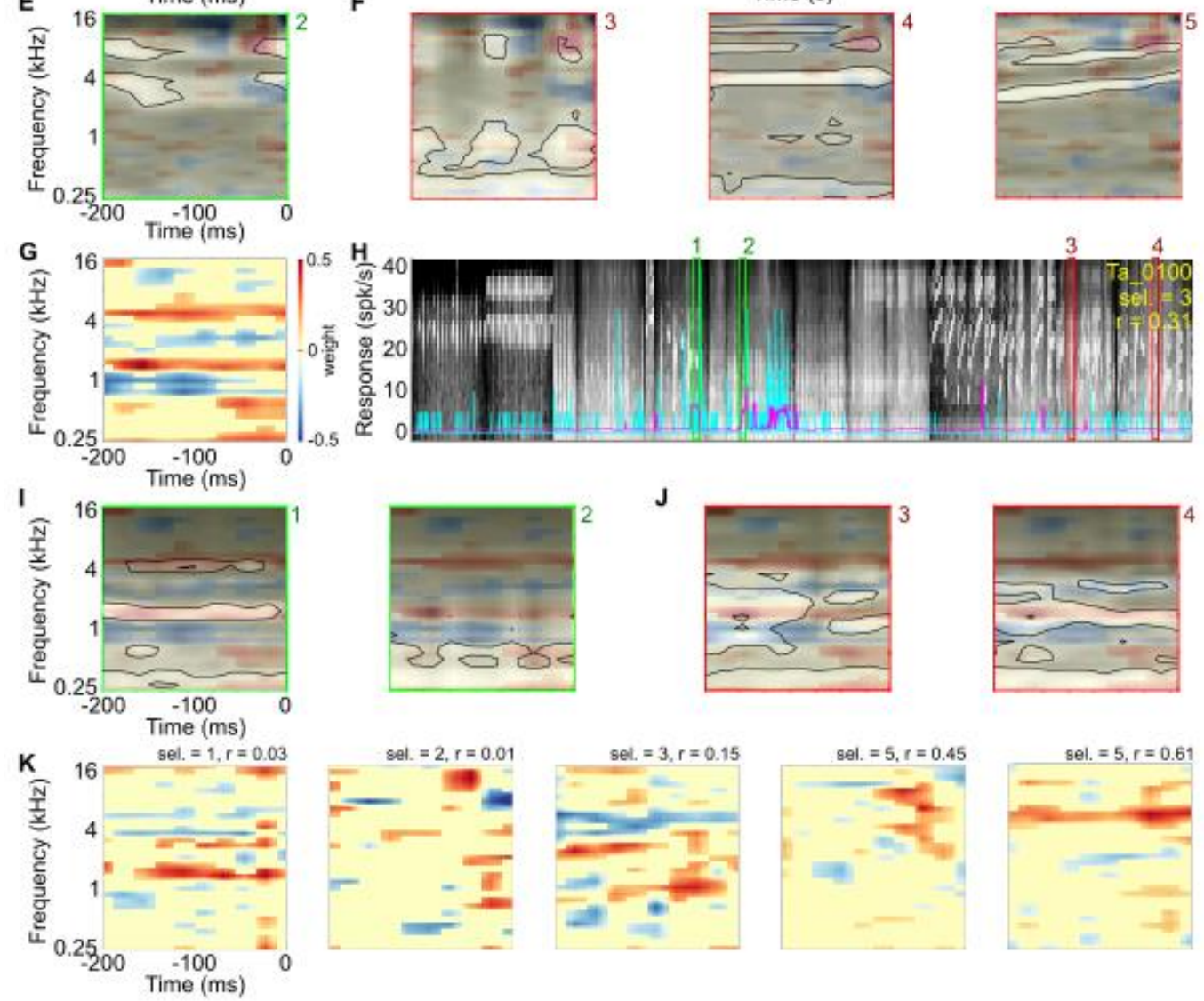

Figure 6: STRF estimates of example A1 L2/3 neurons.

(A, G) STRF estimates of two A1 L2/3 neurons showing complex feature selectivity. (B, H) Stimulus cochleagram (background) and comparison of predicted PSTHs (magenta) and observed responses (cyan) of these two neurons. (D) Expanded cochleagram segment from

395 orange box in B. In B, D, and H, green boxes labeled ' 1 ' and ' 2 ' correspond to $200 \mathrm{~ms}$ long stimulus segments that elicited neural responses. Red boxes labeled ' 3 ', '4', and '5' correspond to $200 \mathrm{~ms}$ long stimulus segments that did not elicit responses. Numbers correspond to examples shown in panels C, E, F, I, and J. (C, E, F) Overlay of stimulus energy in $200 \mathrm{~ms}$ long segments corresponding to numbers in $B$ and $D$ (transparency denotes stimulus energy, peak energy is

400 bounded by black contour) on the STRF (colormap) of this unit. (I, J) Similar to C, E, and F but for the other A1 L2/3 example. (K) Additional examples of complex STRFs of A1 L2/3 neurons. 
For example, the unit in Fig. 6A-F showed selective responses to teeth chatter calls, a non-voiced call which contains repetitive pulses of low-frequency energy around $1 \mathrm{kHz}$ accompanied by high-frequency energy around $8 \mathrm{kHz}$ (see spectrogram in Fig. 2B). The STRF 405 estimate of this neuron showed excitatory receptive field subunits at $\sim 1 \mathrm{kHz}$ and $\sim 8 \mathrm{kHz}$, with an additional excitatory subunit at $8 \mathrm{kHz}$ occurring $\sim 100 \mathrm{~ms}$ later. Some parts of teeth chatter calls thus closely overlapped the excitatory subunits of STRF, driving strong responses (Fig. 6C). But other parts of teeth chatter calls did not drive responses (Fig. 6F), possibly because of the faster repetition rate of individual syllables or activity-dependent adaptation of spiking activity. A second

410 call exemplar that had repetitive energy at $8 \mathrm{kHz}$ (a chirp call) also drove responses in this neuron to a lesser extent (Fig. 6E), but other vocalizations with $8 \mathrm{kHz}$ energy that did not have a repetitive structure did not drive responses (e.g. wheek calls, Fig. 6F). A second example unit that required the presence of harmonic structure is shown in Fig. 6G-J. This unit appeared to require at least two of the excitatory STRF subunits to be activated to produce a response. The selectivity for

415 multiple frequency components in this unit was reminiscent of 'harmonic template neurons' that have been reported in marmoset auditory cortex [45]. This unit responded even when different frequency combinations were excited by different calls (Fig. 6I), underscoring the intuition that these units could not be described as a simple spectral filter. Figure $6 \mathrm{~K}$ shows further examples of STRF estimates of units that showed selective responses to call features.

Over the population of neurons, we did not find significant differences in the performance of the $L N$ models to fit training data segments from vMGB, $A 1 L 4$, or $A 1 L / 3$ neurons (Fig. 7A, left; $p=0.684$, Kruskal-Wallis test), suggesting that the model converged to a solution similarly across the three processing stages. However, while the LN models generalized to the validation data 425 segments with similar performance in VMGB and A1 L4 neurons, generalization was significantly worse for A1 L2/3 neurons (Fig. 7A, right; Kruskal-Wallis test, $p=0.0003$; Dunn-Sidak posthoc test $p$-values are: vMGB vs. A1 L4: $p=0.999, A 1$ L2/3 vs. vMGB: $p=0.003, A 1$ L2/3 vs. A1 L4: $p$ $=0.0006$ ). Critically, model generalization performance was correlated with call selectivity across all processing stages (Fig. 7B; ANOCOVA with selectivity as covariate; $p=2.83 \times 10^{-7}$ ). We note 430 that several neurons with a call-selectivity of 1 showed very low and non-significant $r$ values. These observations suggest that more complex and nonlinear models may be required to capture these highly selective responses. 


\section{Figure 7}
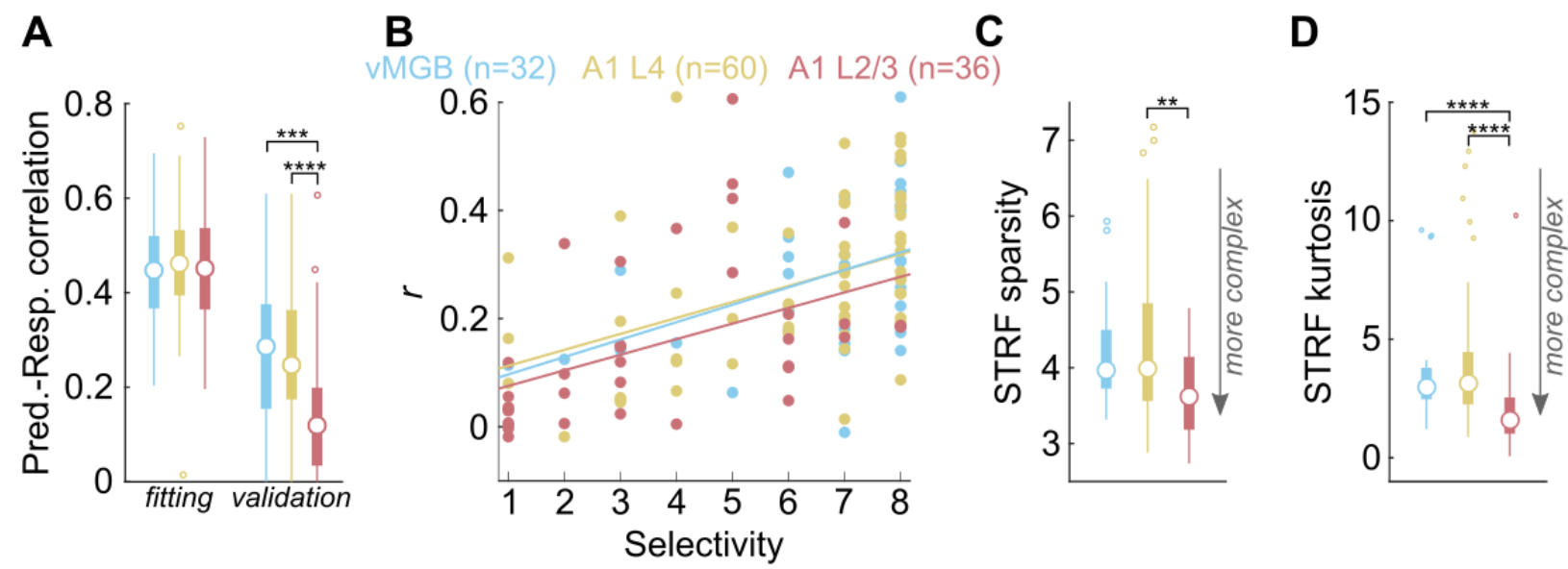

435 Figure 7: Performance and complexity of STRF estimates across processing stages.

(A) Performance of LN models on test and validation data from MGB (blue), A1 L4 (yellow), and A1 L2/3 (red). Discs denote medians, thick lines denote interquartile range, and thin lines correspond to the extent of the distribution. Outliers are shown as dots. (B) Model validation performance plotted as a function of call selectivity across processing stages. Dots are individual

440 neurons and lines correspond to linear fit. (C) Distributions of STRF sparsity across processing stages. Colors and symbols as earlier. (D) Distributions of STRF kurtosis across processing stages. Colors and symbols as earlier. For all panels except B, Kruskal-Wallis tests with posthoc Dunn-Sidak tests were used for statistical comparisons. For B, an ANCOVA with selectivity as a covariate was used. Asterisks correspond to: ${ }^{*}: p<0.05,{ }^{* *}: p<0.01,{ }^{* * *}: p<0.005,{ }^{* * * *}: p<0.001$

445 (exact $p$-values in main text).

We used two metrics to compare the complexity of STRF structure across processing stages. First, we used STRF sparsity, defined as the maximum absolute value of the significancemasked STRF divided by the standard deviation of the significance-masked STRF $[46,47]$. For 'simple' STRFs, the maximum value would be high, whereas standard deviation would be low, resulting in high STRF sparsity values. For complex STRFs where many weight values are large, the maximum value and standard deviation would be comparable, resulting in lower STRF

455 sparsity values. We found a significant effect of processing stage on STRF sparsity (Fig. 7C; Kruskal-Wallis test, $p=0.008$ ), with post-hoc tests revealing a significant difference between $A 1$ L2/3 and A1 L4 neurons (Dunn-Sidak posthoc test, $p=0.006$ ). As a second metric, we quantified the kurtosis of STRF weight values (after significance masking). STRFs with simple structure would show weight distributions with high kurtosis, with most of the weights concentrated in one

460 or two subunits, and the rest of the weights equaling zero. Complex STRFs would be expected to have a more normal distribution of weight values. We found a significant effect of processing stage on kurtosis (Fig. 7D; Kruskal-Wallis test, $p=3.3 \times 10^{-5}$ ), with Dunn-Sidak posthoc tests revealing 
significant differences between A1 L2/3 and vMGB ( $p=0.0007)$ as well as between A1 L2/3 and A1 L4 ( $p=0.0001)$. These statistical results were qualitatively unchanged even when neurons 465 with non-significant $r$ values were excluded. These observations supported our hypothesis that whereas VMGB and A1 L4 neurons responded to call stimuli in a manner that was largely consistent with their spectral tuning properties, A1 L2/3 neurons were driven by more complex spectrotemporal features present in calls that could not be well fit by linear models.

470 Emergence of call feature selectivity in A1 L2/3 confers high stimulus-specific information on to individual A1 L2/3 neural responses

While our data show that $\mathrm{A} 1 \mathrm{~L} 2 / 3$ neurons become call-selective by restricting their responses to specific call features, the consequence of this emergence of call selectivity on decoding call identity from A1 L2/3 neural activity is unclear. An obvious expectation would be that increasing

475 the feature selectivity of single neurons would result in unique activity patterns in response to some calls, thereby leading to higher information carried by these neurons about call identity. Conventionally, mutual information (MI) [48] has been used to estimate the amount of information about stimulus identity carried by neural responses [49-52]. Intuitively, for our call stimulus set consisting of 16 calls, a neuron that exhibits 16 unique response patterns, each corresponding to 480 a call, would provide the maximal $\mathrm{Ml}$ about the stimulus set (in this case, 4 bits of information). When we computed the average $\mathrm{Ml}$ in $100 \mathrm{~ms}$ time bins (50 ms slide; see Methods) of the population of A1 L4 neurons as has been done in most earlier studies [49-52], we found low information levels throughout the response duration (Fig. 8A, yellow) that were not significantly different (two-sided t-test with FDR correction at each time point) from population MI present in

485 the vMGB population (Fig. 8A, blue). However, consistent with a recent result showing decreasing information content in the ascending auditory pathway of anesthetized GPs [53], we found significantly lower MI levels in the A1 L2/3 population (Fig. 8A, red). We confirmed that this result held over a wide range of window sizes used for analysis (Supplementary Fig. 1A). We also found that compared to the VMGB and A1 L4 populations, the A1 L2/3 population displayed longer

490 timescales of integration. When we determined the total population Ml over the entire stimulus duration by integrating the area under the population $\mathrm{Ml}$ curve at each analysis time-bin size used, we found that the population MI in VMGB and A1 L4 saturated at a window size of $200 \mathrm{~ms}$ (100 ms slide), but the population MI in A1 L2/3 did not saturate even at the largest time bin considered (Fig. 8B).This difference in integration time scale paralleled our observations of STRF complexity 495 - compared to VMGB and A1 L4 neurons, A1 L2/3 STRFs were extended in frequency as well as time. 
To understand how lower population Ml levels might arise and to test whether this negatively impacted stimulus decodability in $A 1$ L2/3, we decomposed how information was distributed across two factors, 1) individual neurons and 2) individual stimuli, in the vMGB, A1 L4, and A1 L2/3 neural populations. First, we examined how MI was distributed over the individual neurons that make up the population average in Fig. 8A. Fig. 8D shows $\mathrm{Ml}$ as a function of time for two example A1 L4 neurons (the same neurons as in Fig. 3B left and center). Although the magnitudes of $\mathrm{Ml}$ are different, the $\mathrm{Ml}$ over time is sustained in both cases, which means that when averaged, the mean $\mathrm{Ml}$ will also be sustained over time (as in Fig. 8A, yellow). In contrast,

505 Fig. 8E shows MI for two example A1 L2/3 neurons (the same neurons as in Fig. 3C left and center). Here, the Ml is close to zero for many time bins, and shows peaks in time bins that are non-overlapping between neurons, which means when averaged, the mean Ml will be at a low value (as in Fig $8 \mathrm{~A}$, red). Second, we decomposed the MI into stimulus-specific information (Issi; [54-56]), which measures how much information about each stimulus is provided by the response.

510 Note that the conventionally-computed $\mathrm{MI}$ is the weighted average of $\mathrm{I}_{\mathrm{SSI}}$ across all stimuli. Figs. $8 \mathrm{~F}-\mathrm{H}$ show the decomposition of the $\mathrm{Ml}$ of the example neurons in Figs. $8 \mathrm{C}-\mathrm{E}$ respectively into the IssI for each call stimulus. In A1 L4 (Fig. 8G), Iss। was evenly distributed across all stimuli and time bins, resulting in the average (the MI; Fig. 8D) being at a sustained level over time. Note that later time bins for some calls (Fig. $8 \mathrm{G}$ left; wheeks and whines) have high information content

515 because these calls are the longest in our stimulus set, and this neuron responded throughout the call durations. In A1 L2/3, however (Fig. 8H), Issı was very high (approaching 3 bits) for specific stimuli only at specific time bins. Thus, average Issı across stimuli, as is done to compute MI (Fig. $8 \mathrm{E})$, approached zero for most time bins, and severely underestimated the informativeness of the response.

To quantify whether a high MI time bin (see Methods; red crosses in Fig. 8C-E) arises from an approximately normal distribution of Issı across all stimuli for that time bin (as in Fig. 8G), or from a highly skewed Issi distribution across stimuli for that time bin (as in Fig. 8H), we computed a MI Sparsity Index ( $S I_{M l}$; see Methods). $S I_{M I}$ increased significantly between all three processing 525 stages tested (Fig. 8I; $p=5.4 \times 10^{-7}$, Kruskal-Wallis test; Dunn-Sidak posthoc test $p$-values are: vMGB vs. A1 L4: $p=0.005, A 1$ L2/3 vs. vMGB: $p=1.6 \times 10^{-5}, A 1$ L2/3 vs. A1 L4: $p=0.015$ ), with A1 L2/3 neurons being informative about only a few calls in their most informative time bins. 

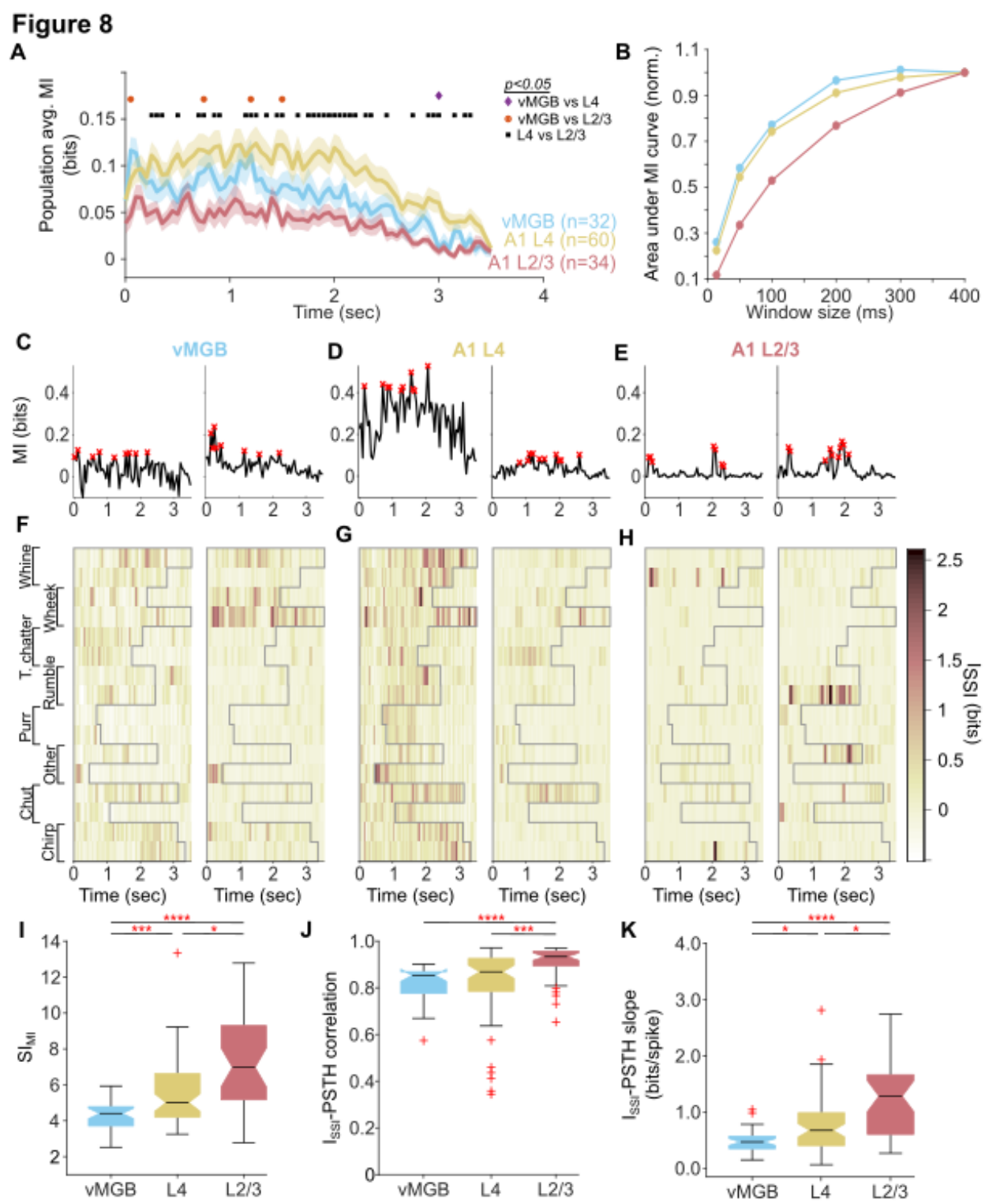

Figure 8: Reformatting of stimulus information in A1 L2/3.

(A) Population average of $\mathrm{Ml}$ as a function of time in VMGB (blue), A1 L4 (yellow), and A1 L2/3 (red) neurons. Lines correspond to means and shading to 1 s.e.m. Colored dots represent results of statistical testing ( $p<0.05$; two-sided t-test with FDR correction for multiple comparisons). (B) Area under the population average MI curves in when time bins of different lengths were used to evaluate MI. vMGB and A1 L4 show similar timescales of temporal integration, whereas A1 L2/3 neurons show longer timescales of integration. (C, D, E) MI for two example neurons each from vMGB (C), A1 L4 (D), and A1 L2/3 (E). The example neurons are the same as the left two examples from Fig. $3 A-C$. Red crosses correspond to high MI time bins. $(\mathbf{F}, \mathbf{G}, \mathbf{H})$ IssI for the vMGB neurons in (C), the A1 L4 neurons in (D), and the A1 L2/3 neurons in (E). Darker colors correspond to higher IssI values. (I) Distributions of $\mathrm{SI}_{\mathrm{MI}}$ for vMGB, A1 L4, and A1 L2/3 neurons. Horizontal line corresponds to median and colored area corresponds to interquartile range. (J) Distributions of IssI - PSTH correlation coefficients for VMGB, A1 L4, and A1 L2/3 neurons. (K) Distributions of IssI - PSTH slopes for vMGB, A1 L,4 and A1 L2/3 neurons. Asterisks correspond to: *: $p<0.05,{ }^{* *}$ : $p<0.01,{ }^{* * *}: p<0.005,{ }^{* * * *}: p<0.001$ (Kruskal-Wallis test with posthoc Dunn-Sidak tests, exact $p-$ values in main text). 
$\mathrm{Ml}$ analysis only takes into account spike patterns, but does not distinguish between the presence or absence of spikes. In other words, if a neuron responds to 15 of the 16 call stimuli, and is inhibited by 1 call, the information provided by this neuron about the stimulus set is equivalent to that provided by a neuron that responds to only one call. To determine whether IsSI is provided by the presence or absence of spikes, we computed the cross-correlation between the PSTH and Iss for neurons in VMGB, A1 L4, and A1 L2/3. Compared to vMGB and A1 L4, A1

$550 \mathrm{~L} 2 / 3$ neurons showed higher $\mathrm{I}_{\mathrm{SSI}}$ - PSTH correlations, suggesting that $\mathrm{A} 1 \mathrm{~L} 2 / 3$ responses were informative because of presence of spikes (Fig. 8J; $p=2.2 \times 10^{-5}$, Kruskal-Wallis test; Dunn-Sidak posthoc test $p$-values are: vMGB vs. A1 L4: $p=0.136, A 1 \mathrm{~L} 2 / 3$ vs. vMGB: $p=1.1 \times 10^{-5}, A 1 \mathrm{~L} 2 / 3$ vs. $A 1 \mathrm{~L} 4: \mathrm{p}=0.003)$. Compared to $A 1 \mathrm{~L} 4$, the $\mathrm{I}_{\mathrm{sI}}-\mathrm{PSTH}$ relationship in $\mathrm{A} 1 \mathrm{~L} 2 / 3$ also showed a significantly higher slope, indicating that each spike from an $A 1 L 2 / 3$ neuron carried greater

555 stimulus-specific information (Fig. 8K; $p=6.2 \times 10^{-6}$, Kruskal-Wallis test; Dunn-Sidak posthoc test p-values are: vMGB vs. A1 L4: $p=0.018, A 1$ L2/3 vs. vMGB: $p=2.9 \times 10^{-6}, A 1$ L2/3 vs. A1 L4: $p$ $=0.016)$. We confirmed that these results were consistent over a wide range of window sizes used for analysis (Supplementary Figs. 1B-D).

Table 1 is a summary of all statistical comparisons of basic tuning properties, selectivity metrics, STRF metrics, and information theoretic metrics of VMGB, A1 L4 and A1 L2/3 neurons. If call selectivity gradually developed over the three processing stages, one would expect to see differences in selectivity parameters pairwise between all three processing stages. In contrast, if selectivity arose de-novo in superficial cortical layers, vMGB and A1 L4 parameter distributions

565 would not be significantly different, but A1 L2/3 and A1 L4 (as well as A1 L2/3 vs. vMGB) would show significant differences. Our results support the latter possibility and the idea that while subcortical activity and inputs to A1 represent vocalizations densely and based on spectral content, a call feature-based representation emerges in A1 L2/3 that dramatically transforms how information about conspecific calls is represented in A1 outputs. 
Table 1: Statistical summary of comparisons between vMGB, A1 L4, and A1 L2/3.

$580 \quad\left({ }^{* * * *}: p<0.001,{ }^{* * *}: p<0.005,{ }^{* *}: p<0.01,{ }^{*}: p<0.05\right.$, n.s.: not significant. All tests are Kruskal-Wallis tests with post-hoc Dunn-Sidak tests unless noted.)

\begin{tabular}{|c|c|c|c|}
\hline Parameter & $\begin{array}{l}\text { vMGB vs. } \\
\text { A1 L4 }\end{array}$ & $\begin{array}{l}\text { A1 L2/3 vs. } \\
\text { vMGB }\end{array}$ & $\begin{array}{l}\text { A1 L2/3 vs. } \\
\text { A1 L4 }\end{array}$ \\
\hline \multicolumn{4}{|l|}{ Basic properties } \\
\hline $\begin{array}{l}\text { Bandwidth } \\
\text { ANCOVA (posthoc Tukey's HSD) }\end{array}$ & $*$ & $* * *$ & n.s. \\
\hline Spontaneous rate & n.s. & $* * *$ & n.s. \\
\hline \multicolumn{4}{|l|}{ Selectivity parameters } \\
\hline $\begin{array}{l}\text { Selectivity } \\
\text { (overall firing rate) }\end{array}$ & n.s. & $* * * *$ & $* * * *$ \\
\hline $\begin{array}{l}\text { Selectivity } \\
\text { (response windows) }\end{array}$ & n.s. & $* * * *$ & $* * * *$ \\
\hline $\begin{array}{l}\text { No. of windows and response length } \\
\text { 2-D K-S (Bonferroni correction) }\end{array}$ & n.s. & $* * * *$ & $* * *$ \\
\hline Kurtosis & n.s. & $* * * *$ & $* * * *$ \\
\hline Activity fraction & n.s. & $* * *$ & $* * *$ \\
\hline \multicolumn{4}{|l|}{ STRF parameters } \\
\hline$r$ & n.s. & $* * *$ & $* * * *$ \\
\hline STRF sparsity & n.s. & n.s. & $* *$ \\
\hline STRF kurtosis & n.s. & $* * * *$ & $* * * *$ \\
\hline \multicolumn{4}{|l|}{$\begin{array}{l}\text { Mutual Info. analyses } \\
\text { (100 ms time bins) }\end{array}$} \\
\hline $\begin{array}{l}\text { Population MI } \\
\text { 2-sided t-test (FDR correction) }\end{array}$ & $\begin{array}{l}\text { very few time } \\
\text { points }\end{array}$ & $\begin{array}{l}\text { few time } \\
\text { points }\end{array}$ & $\begin{array}{l}\text { many time } \\
\text { points }\end{array}$ \\
\hline $\mathrm{SI}_{\mathrm{MI}}$ & $* * *$ & $* * * *$ & $* *$ \\
\hline ISSI-PSTH correlation & n.s. & $* * * *$ & $* * *$ \\
\hline ISSI-PSTH slope & $*$ & $* * * *$ & $*$ \\
\hline
\end{tabular}




\section{Discussion}

Although many previous studies have explored the neural representation of conspecific

585 calls in subcortical and cortical areas across species [6-9, 15-27, 57], exactly where and how call selective responses emerge in the auditory processing hierarchy has remained unclear. In mice, some studies have suggested that selectivity for ultrasonic vocalizations (USVs) in a manner not consistent with spectral content might arise at subcortical stations [5], and lead to an over-representation of USV-selective responses in the IC [58]. However, other studies have

590 suggested that this over-representation is explained by a tonotopic expansion of the representation of those frequencies, and that USV responses are in fact consistent with spectral tuning of neurons [59]. In bats, the majority of neurons in subcortical processing stations responded to calls consistent with neurons' frequency tuning [3,60]. In GPs, single neurons in the IC are not selective for particular call types or call features [16]. In the MGB, although single

595 neurons follow call envelopes less precisely [15] and neural responses to calls are less predictable from neurons' tone tuning [61], responses do not differentiate between natural and reversed versions of calls [62], suggesting that MGB responses are not call or call feature selective. At the level of $A 1$, some studies have reported that single neurons show selectivity for natural calls over reversed calls [18], or that neurons seem to respond to calls that share similar

600 spectrotemporal features [23], but by and large, neural responses to calls seem to be explained by the frequency tuning of neurons [7, 21]. At the level of secondary cortex, neurons have been shown to be highly selective for call type in primates [8, 9] and GPs (Area S and VRB [6]). However, because of some technical limitations of these studies, including the use of anesthesia, limited stimulus sets, multi-unit recordings, or not comparing across processing stages, 605 specifically across cortical laminae, it is difficult to evaluate where transformations to call representation begin to occur. Answering the 'where' question is a critical first step that will enable the targeting of experiments probing the neural mechanisms underlying these transformations to the appropriate target processing stage. In this study, we overcame these limitations by simultaneously: 1) conducting experiments in unanesthetized animals, 2) using an extensive set

610 of conspecific calls as stimuli, 3) comparing across thalamic and cortical processing stages, and 4) separating $A 1$ neurons recorded from thalamorecipient and superficial layers. We found that whereas call representations in VMGB and A1 L4 were similar, a critical transformation occurs between A1 L4 and A1 L2/3. While VMGB and A1 L4 neurons seemed to respond primarily to the spectral content of calls resulting in a dense representation of calls, many A1 L2/3 responses

615 were contingent on the presence of specific spectrotemporal features, resulting in a highly sparse representation of calls. 
This observed transformation is consistent with previously reported increases in the nonlinearity of neural receptive fields in marmosets [31], increases in sparsity of responses in rats [63], and some reports of increased receptive field complexity in superficial A1 layers (in cats [64620 66]). This transformation is also consistent with ultra-high-field human $\mathrm{fMRI}$ studies showing that supragranular BOLD responses are less readily explained using simple frequency tuning models [33]. Thus, the transformation of sound representation between $A 1 L 4$ and $A 1 L 2 / 3$ appears to be a conserved phenomenon across species, from GPs to humans. In non-human primates, secondary auditory cortical areas have been shown to exhibit call-selective responses [8, 9], and

625 the highest sensory cortical regions of the auditory processing pathway preferentially represent conspecific calls [10-12]. Our results suggest that the emergence of call feature-selectivity at supragranular A1 layers is a critical first step in building call-selective cortical specializations.

How could highly feature-selective neurons be generated? In an earlier study in marmosets, many A1 neurons recorded at shallow cortical depths were combination-selective, i.e., these neurons showed responses only when specific frequencies were present with precise temporal relationships [31]. Such nonlinear mechanisms could generate call feature-selectivity, but how precise temporal delays necessary for this computation are generated in the A1 circuit remains an open question. A second possibility is that although A1 L4 neurons are not selective

635 for call type in that they respond to all categories, responses to some calls in a subset of time bins may be marginally stronger (Fig. 9A, B). Pooling a number of A1 L4 neurons that exhibit similar marginally stronger responses to the same time bin, but whose responses are uncorrelated otherwise, could accentuate the differences between this preferred time bin and other bins. The higher $\mathrm{SI}_{\mathrm{MI}}$ observed in A1 L4 neurons compared to VMGB neurons supports the notion that there

640 may be local periods of high information in the A1 L4 population responses. Applying a strong nonlinearity to these pooled inputs could in principle create $A 1 L 2 / 3$ responses that are highly selective for particular spectrotemporal call features (Fig. 9C, D). Supporting the notion that A1 $L 2 / 3$ neurons might be applying high thresholds is the fact that $A 1 L 2 / 3$ neurons are known to exhibit very low spontaneous rates across species [31,63], including in our own data (Fig. 1E).

Extending this model to the next linear (pooling) stage, the responses of multiple featureselective A1 L2/3 neurons that respond to features belonging to the same call category could be integrated by neurons in secondary cortical areas to result in sustained call category-selective responses. In anesthetized GPs, neurons that show dense firing with high contrast between call 650 categories, which is highly useful in discriminating between call categories, have been reported 
in secondary areas VRB and S [6]. It is yet to be determined whether additional mechanisms could be used to increase call category selectivity by further restricting responses to only if call features are detected in a particular temporal sequence, which for example could be achieved by some forms of dendritic computation [67, 68, 69]. Our proposed model is based on model

655 architectures with alternating linear and nonlinear stages that have been used to explain responses in inferotemporal cortex [70]. These models are based on exclusively excitatory and feedforward operations. Other models, for example, incorporating recurrent excitatory inputs that have been shown to sharpen cortical tuning [71], or those involving cortical inhibition which could also fine-tune cortical selectivity [72-75], represent alternative architectures that are more 660 complex but biologically realistic. Specific cortical inhibitory cell types, for example, somatostatinexpressing interneurons, might play a role in generating sharp frequency tuning [76]. Thus, extensive theoretical and experimental work is necessary to test these models and dissect the neural mechanisms underlying the generation of feature selectivity.

\section{Figure 9}

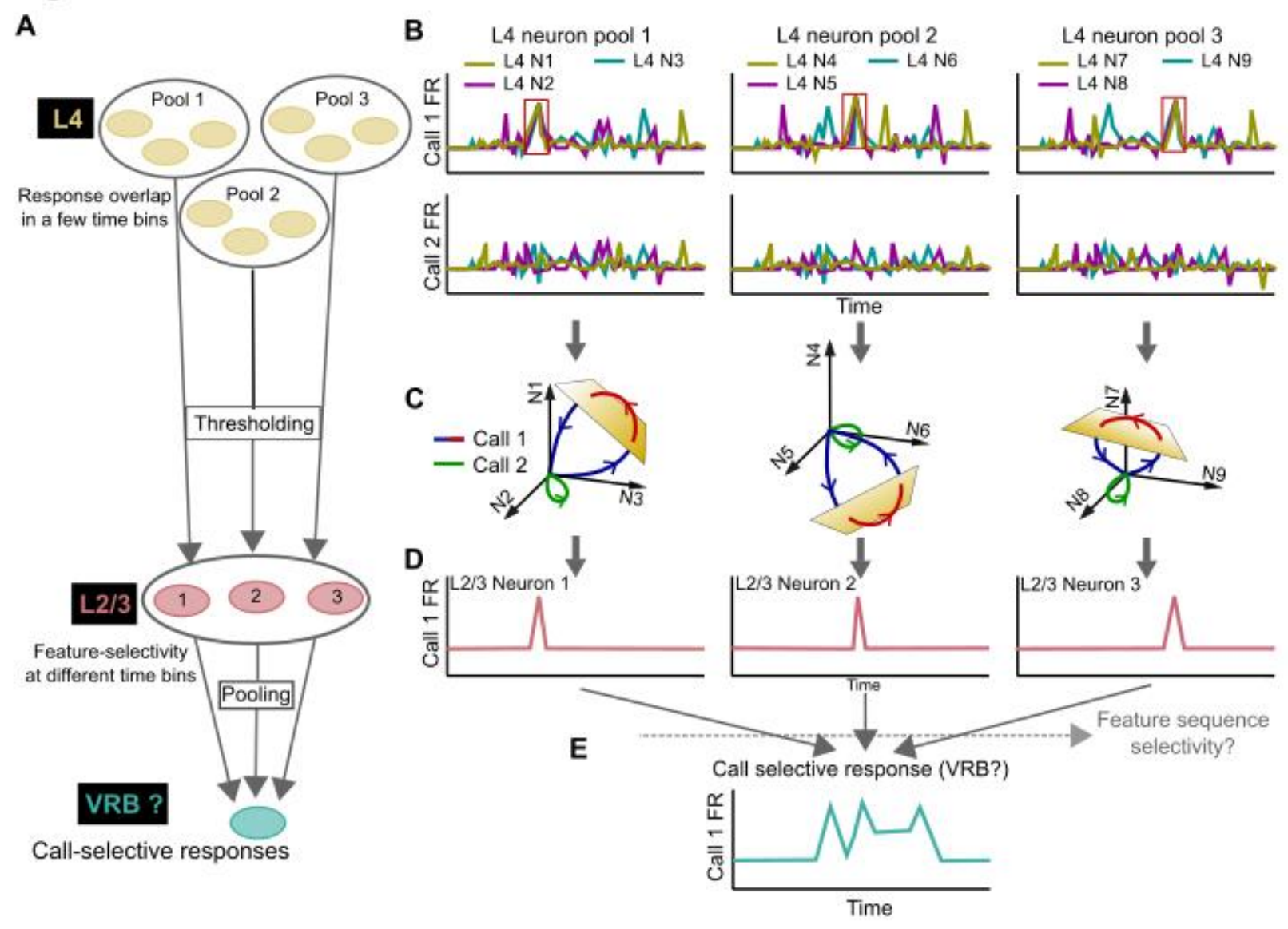

665 Figure 9: Working model of generating call-selectivity in the auditory cortical hierarchy. (A) Alternating nonlinear (high threshold) and more linear (pooling) stages that could result in callselective responses in secondary cortex. (B) Schematic of non-selective A1 L4 neural responses 
that could show overlap in a few time bins. (C, D) A high threshold could be applied to different pools of $\mathrm{A} 1 \mathrm{~L} 4$ neurons to result in $\mathrm{A} 1 \mathrm{~L} 2 / 3$ responses that are selective to specific call features. (E) A more linear operation could pool over A1 L2/3 neurons that are selective for features belonging to the same call category to result in call category-selective secondary cortical responses.

What could be the advantages of a highly sparse representation? Extensive work in the visual cortex has proposed that sparse coding could allow for increased storage capacity for associative memory, is more energy efficient, and could make read out by downstream areas easier [77]. The possibility of easier readout is especially interesting in the auditory system, where highly variable continuous inputs need to be parsed and sequenced into categorical units (for

680 example, words in human speech or call category in animal communication calls). The 'dense' codes we found in VMGB and A1 L4 are redundant to some degree because neurons respond to highly overlapping stimulus sets. Thus, the activity of a single neuron in A1 L4 signaled the presence of multiple call features, with the actual feature identity being encoded over the population. This is reflected in our information theoretic analysis showing that in A1 L4, mutual

685 information is distributed both over time bins and over neurons. A1 L2/3 effectively decorrelated A1 L4 activity, so that single neurons now carried high levels of information about the stimulus. One consequence of this decorrelation is an increase in the dimensionality of sound representation, which could serve to 'untangle' [78] highly variable representations of different sound categories. As mentioned earlier, in a further processing step, a linear pooling operation

690 could be used to pool responses of A1 L2/3 neurons that respond to different features of the same call type, resulting in truly call category-selective responses such as those observed in secondary cortical areas [8, 9]. Further analysis is necessary to quantify the dimensionality of sound representation in different cortical layers and the separability of different call categories. In the auditory system, a second consideration for a neural code is robustness to environmental noise

695 - realistic listening conditions add reverberations, noise, and competing sounds to the target sound impinging on our ears. It remains to be seen whether the feature-selective responses we have observed in A1 L2/3 neurons will remain invariant to these perturbations, and will provide a more robust representation of sounds than the dense representations in A1 L4.

In conclusion, by recording from successive auditory processing stages in awake animals using a rich and behaviorally-relevant stimulus set, we have demonstrated that rather than a gradual emergence of feature selectivity over the auditory processing hierarchy, selectivity for sound features appears to emerge de-novo in the superficial layers of auditory cortex, resulting 
705 transformations to sound representations occur at the superficial layers of $A 1$. These data set the stage for further studies investigating the biophysical and circuit mechanisms by which call feature selectivity arises from non-selective inputs, and how these feature-selective responses could be read-out by downstream call category-selective neurons. Our data suggest that the root of observed cortical specializations for call processing [10-12] could in fact reside in primary auditory

710 cortex.

\section{Materials and Methods}

\section{Ethics}

All experimental procedures conformed to NIH Guide for the care and use of laboratory animals,

715 and were approved by the Institutional Animal Care and Use Committee (IACUC) of the University of Pittsburgh.

\section{Animals}

We acquired data from 4 male and 2 female adult, wild-type, pigmented guinea pigs (Cavia 720 porcellus; Elm Hill Labs, Chelmsford, MA), weighing $\sim 600-1000 \mathrm{~g}$ over the course of the experiments.

\section{Surgical procedures}

All experiments were conducted in unanesthetized, head-fixed, passively-listening animals. To

725 achieve head fixation, a custom head post was first surgically anchored onto the skull using dental acrylic (Metabond, Parkell Inc.) following aseptic techniques under isoflurane anesthesia. Chambers for electrophysiological recordings were positioned over the location of auditory cortex using anatomical landmarks [6, 36, 37]. Post-surgical care, including administration of systemic and topical analgesics, was provided for 3 - 5 days. Following a 2-week recovery period, animals

730 were gradually adapted to the experimental setup by slowly increasing the duration of head fixation.

\section{Acoustic stimuli}

All stimuli were generated in Matlab (Mathworks, Inc.) at a sampling rate of $100 \mathrm{kHz}$, converted 735 to analog (National Instruments), attenuated (TDT), power-amplified (TDT), and delivered through a speaker (TangBand) located $\sim 90 \mathrm{~cm}$ from the animal on the contralateral side. We used a wide variety of stimuli including pure tones, noise bursts, frequency- and amplitude-modulated sounds, 
two-tone pips, and conspecific vocalizations as search stimuli to initially detect and isolate single units. Once we isolated a unit, we delivered pure tones (50 or $100 \mathrm{~ms}$ ) covering 7 octaves in

740 frequency $(200 \mathrm{~Hz}-25.6 \mathrm{kHz}, 10$ steps/oct.) at different sound levels (20 dB SPL spacing) to characterize its frequency response area. We defined the best frequency of the unit as the frequency eliciting the highest firing rate, best level as the sound level eliciting the highest firing rate. The bandwidth of the unit was estimated using a rectangle fit to the frequency tuning curve at the best level [79]. After characterizing basic tuning properties, we presented conspecific

745 vocalization stimuli. All vocalizations were recorded in our animal colony using Sound Analysis Pro [80] by placing one or more animals in a sound-attenuated booth and by recording vocalizations using a directional microphone (Behringer). Two observers manually segmented and classified vocalizations into categories based on previously published criteria [6, 34, 35]. We verified high inter-observer reliability using Cohen's Kappa statistic $(\kappa=0.8)$. In

750 electrophysiological experiments, we typically presented 2 exemplars each of 8 vocalization categories (16 vocalization stimuli; 0.4 - 3.5 s length depending on call type; typically, 10 repetitions of each stimulus). For some units, we presented additional exemplars belonging to some categories (24 stimuli), but only presented 5 repetitions. All vocalizations were normalized for r.m.s. power and presented at $70 \mathrm{~dB}$ SPL in random order, with a random inter-trial interval

755 between 2 and 3 seconds. For some units, we also presented vocalizations to which we added reverberations or noise (not presented in the current manuscript).

\section{Electrophysiology}

All recordings were conducted in a sound-attenuated booth (IAC) whose walls were lined with 760 anechoic foam (Pinta Acoustics). Animals were head-fixed in a custom acrylic enclosure affixed to a vibration-isolation tabletop that provided loose restraint of the body. We recorded the activity of single units in the ventral medial geniculate body (VMGB) and identified cortical laminae of primary auditory cortex (A1). We sequentially performed small craniotomies ( 1 mm dia.) within the recording chamber using a dental drill (Osada) attached to a stereotactic manipulator (Kopf)

765 to reach regions of interest. For vMGB recordings, we targeted previously published stereotactic coordinates [81, 82] by performing a caudally-angled craniotomy in the caudal part of the chamber. The location of the electrode in the vMGB was confirmed using electrophysiological properties (strong tone responses, low response latency, and expected tonotopic organization $[83,84])$. For cortical recordings, we performed craniotomies over the expected anatomical

770 location of $\mathrm{A} 1[6,36,37]$ angled to be roughly perpendicular to the cortical surface. We used strong tone responses and tonotopic reversals to confirm that the recording location was within 
A1. In each recording session, we used a hydraulic microdrive (FHC) to advance a tungsten microelectrode (FHC or A-M Systems; 2 - $5 \mathrm{M} \Omega$ impedance) through the dura into the underlying target tissue. Electrophysiological signals were digitized and amplified using a low-noise amplifier

775 (Ripple Scout), and data visualized online (Trellis software suite). We played a wide variety of search stimuli while slowly advancing the electrode. When a putative spike was detected, we used a template-matching algorithm for online spike-sorting to isolate single units. Sorting was further refined offline at the conclusion of the experimental session (MKSort). Using this technique, we typically acquired spike data from $1-3$ single units simultaneously. Spike

780 waveforms were classified into putative regular-spiking (RS) and fast-spiking (FS) categories using the peak-to-trough ratio and spike width as parameters. We only considered well-isolated single units, defined as having a peak amplitude at least 5.5 standard deviations above noise baseline, for further analysis. For A1 recordings, we sequentially recorded neural activity from superficial to deep cortical layers. At the end of each electrode track, we advanced the electrode

785 to a depth of $\sim 2 \mathrm{~mm}$, and acquired LFP responses every $100 \mu \mathrm{m}$ while retracting the electrode. To do so, we presented 100 repetitions of a pure tone at $70 \mathrm{~dB}$ SPL, with pure tone frequency chosen to match the best frequency of the recorded column. From these local potential data, we calculated the current source density (CSD) defined as the second spatial derivative of the LFP, based on which we assigned recorded units to thalamorecipient or superficial layers [38]. After

790 the electrode was completely retracted, the craniotomy was filled with antibiotic ointment, and recording chambers sealed using a silicone polymer (KwikSil or similar). Recording sessions were limited to 4 hours, and we typically recorded from each craniotomy for $4-8$ electrode tracks. Craniotomies were sealed with dental cement after data acquisition was completed.

\section{Data analysis and statistics}

Analysis was based on data from 45 L2/3 RS neurons, 67 L4 RS neurons, and 33 vMGB neurons that responded to at least one vocalization in our stimulus set. We also isolated 10 call-responsive FS neurons from A1 recordings, which were not analyzed in this study.

800 Response window analysis: We obtained response rate estimates limited to small time bins using an algorithm similar to Issa \& Wang (2008) [85] (also see [86-88]). Briefly, we started with seed windows selected using relaxed criteria and gradually added additional windows until the final window met stringent criteria. To do so, we first determined whether the responses to any call in any $100 \mathrm{~ms}$ window (50 ms slide) located from $50 \mathrm{~ms}$ post stimulus onset until $100 \mathrm{~ms}$ post 805 stimulus offset met two criteria: 1) the average rate exceeded 6 s.e.m. of the spontaneous rate 
and 2) the trial-wise response distribution within the window was significantly different from the spontaneous response distribution with $p_{\text {soft }} \leq 0.1$ (single-tailed t-test with false discovery rate (FDR) correction; this test is used for determining all $p$-values for response window analysis). The initial window could then grow in either direction by adding neighboring windows, if: 1) the 810 response in window to be added met a threshold of $p_{\text {soft }} \leq 0.1,2$ ) average rate in the enlarged window exceeded 10 s.e.m. of the spontaneous rate and 3) the trial-wise response distribution within the enlarged window met a threshold of $p_{\text {add }} \leq 0.01$. We successively added response segments until these thresholds could not be met. To avoid a single bursty trial from spuriously increasing response rate, we replaced trial-wise rates with a z-score $>1.96$ by the mean response

815 rate of the enlarged window. The resultant window was considered the final response window if: 1) the average rate exceeded 14 s.e.m. of the spontaneous rate, 2) the trial-wise response distribution within the final window met a threshold of $p_{\text {final }} \leq 0.0001$, and 3 ) if responses were present on at least $60 \%$ of the trials. Any windows less than $100 \mathrm{~ms}$ apart were coalesced if the resulting window still met the three final stringent criteria. If no response windows were detected

820 for any call, we relaxed the following parameters in order: minimum trial threshold decreased to $50 \%$, z-score for burst detection increased to 2.5 , and window length increased to $200 \mathrm{~ms}$ (slide $=100 \mathrm{~ms}$ ). For example, minimum responsive trial threshold was decreased to $50 \%$ and burst detection z-score increased to 2.5 for the neuron in Fig. 3C (right). Parameters for automated response window analysis were initially chosen to broadly match response regions to visual

825 judgements of three independent observers in a small sample of neurons from the three processing stages. Results were verified to be largely consistent over a range of parameter values. While this automated analysis reliably detected excitatory responses, because of the very low spontaneous rates of cortical neurons, inhibitory responses could not be captured. Thus, when responses were mainly inhibitory rather than excitatory ( 2 neurons in A1 L2/3 and 9 neurons

830 in A1 L4), the number of calls with significant responses was determined manually by three independent observers.

Quantification of selective responses: We quantified the selectivity of neural responses based on the following metrics. 1) We defined call selectivity as the number of call categories with significant 835 responses - if at least one response window was detected for any exemplar belonging to a category, we counted the neuron as being responsive to that category. 2) The number of response windows per call. 3) The length of the response, which was the sum of all window lengths within a call, expressed as a fraction of the total length of that call. Together, metrics (2) and (3) indicated if a neuron was feature-selective - for highly feature-selective neurons, we observed a small 
840 number of short windows, whereas for neurons with low selectivity, we observed many short windows or a single long window. We compared selectivity across processing stages using Kruskal-Wallis tests followed by pairwise post-hoc tests. To quantify differences in feature selectivity across processing stages, we constructed two-dimensional distributions of the number of windows versus window length, and evaluated significance using two-dimensional KS tests 845 [38] with Bonferroni correction.

Sparsity: We estimated sparsity using two metrics: 1) As the reduced kurtosis of trial-wise firing rate responses $[79,89]$, computed over a single window from $50 \mathrm{~ms}$ after stimulus onset to 100 ms after stimulus offset. A reduced kurtosis of zero indicates a normal distribution of firing rates 850 across stimuli or response bins, suggesting a response that is not feature selective. High kurtosis values arise when many response rates are zero and few response rates are high, suggesting highly feature-selective responses. 2) As the activity fraction [40, 41], defined as:

$A=\frac{\left[\sum_{i=1}^{N} r_{i} / N\right]^{2}}{\sum_{i=1}^{N}\left[r_{i}^{2} / N\right]}$

An activity fraction close to zero signifies highly sparse responses, and activity fraction close to 855 one signifies dense responses. Sparsity across processing stages was compared using KruskalWallis tests followed by pairwise post-hoc Dunn-Sidak tests.

Receptive field models: We used the Neural Encoding Model System (NEMS; [42,43]; https://github.com/LBHB/NEMS) as a platform to build linear-nonlinear models and estimate

860 STRFs of call-responsive neurons. The input to the model consisted of the cochleagram of all call stimuli concatenated in time. To compute the cochleagram, we used a fast approximation algorithm that used weighted log-spaced frequency bins and three rate-level transformations corresponding to three categories of auditory nerve fibers ([44]; https://github.com/monzilur/cochlear_models). Previous work has shown that this transformation 865 can adequately capture the inputs to auditory cortex [44]. The resolution of the cochleagram was set at 5 steps/oct. in frequency (total 6 oct. spanning $250 \mathrm{~Hz}-16 \mathrm{kHz}$ ) and $20 \mathrm{~ms}$ in time. Linear weights and the parameters of a point nonlinearity (double exponential function) were estimated by gradient descent to minimize the squared error between the predicted PSTH and the actual PSTH (computed in 20 ms bins, averaged over 10 repetitions). The matrix of linear weights was 870 taken to represent the receptive field, or STRF of the neuron. We performed a nested crossvalidation, where for every neuron's call responses, we used $90 \%$ of the data to fit the models and the remaining $10 \%$ to validate the models. This procedure was repeated 10 times using non- 
875 responses was used as a metric of goodness-of-fit. A bootstrap procedure was used to test for significance of $r$ values. For quantifying STRF complexity and display, we used the mean STRF (over the 10 cross-validation runs) multiplied by a significance mask. To estimate the mask, we used a bootstrap procedure by scrambling the actual linear weight matrices 1000 times to estimate the distribution of weights at each time and frequency bin, and used a two-tailed

880 permutation test to evaluate if the observed STRF mean weights differed significantly (using FDR correction for 310 comparisons) from the bootstrap distributions. To quantify the complexity of STRFs, we used STRF sparsity [46, 47], defined as the maximum absolute value of the significance-masked STRF divided by the standard deviation of the significance-masked STRF, and as a second metric, the kurtosis of significance-masked STRF weights. Sparsity and kurtosis 885 across processing stages were compared using Kruskal-Wallis tests followed by Dunn-Sidak posthoc tests.

Information theoretic analyses: We used stimulus-specific information (IssI) [54-56] to estimate the amount of information that each recorded neuron provided about each stimulus. We also 890 computed the weighted average of Issı across stimuli to determine overall information content, which is conventionally referred to as the mutual information (MI) between the stimulus and response. Only neurons that had completed 10 trials for all the stimuli were considered for this analysis. Intuitively, if a neuron shows a consistent response pattern to a given stimulus, then it has high $I_{\text {ss }}$ about that stimulus. To quantify $I_{S S I}$ we extracted responses beginning 50 ms before

895 stimulus onset and lasting until $50 \mathrm{~ms}$ after the length of the longest stimulus [49] in windows of varying lengths $(14,50,100,200,300$ and $400 \mathrm{~ms}$ with slide equal to half the window size). For shorter duration calls, we populated time bins occurring at times greater than one second after stimulus offset with simulated spikes, with spike rate set at the average spontaneous rate of the neuron. For each window size, the $I_{s s ı}$ in each time bin was calculated as:

$$
I_{S S I}=\sum_{r e s p} p(\text { resp } \mid s t i m) * I_{S P}(\text { resp })
$$

where $I_{S P}(r e s p)$ is the information conveyed by a specific response pattern, calculated as:

$$
\begin{aligned}
& I_{S P}(\text { resp })=\text { TotalEntropy }- \text { ConditionalEntropy }(\text { resp }) \\
& \text { TotalEntropy }=-\sum_{\text {stim }} p(\text { stim }) * \log _{2}(p(\text { stim }))
\end{aligned}
$$


To correct for estimation bias arising from finite trial numbers that likely undersample response probability distributions, we subtracted an all-way shuffled estimate of Issı (average of 100 randomizations [56]) from the value of IssI estimated earlier. All reported values refer to the bias-

910 corrected IssI estimate.

Having obtained these $I_{S S I}$ estimates, we computed how $I_{S S I}$ values were distributed across time bins and across stimuli, and how $I_{S S I}$ correlated with spiking responses of each neuron. To quantify how IssI values were distributed across time bins and across stimuli, for each window size, we

915 calculated a MI sparsity index $\left(S I_{M I}\right.$, defined as the mean kurtosis of $I_{S S I}$ values in high-MI time bins, with high-MI bins defined as bins with MI values exceeding 1 standard deviation of the MI values across all time bins. To determine whether high Issı resulted from the presence or absence of spiking, we calculated the correlation between the Issı and PSTH. Finally, to determine how much information was conveyed by each spike, we determined the slope of the ISSI $_{\text {VS. PSTH }}$

920 distribution. Distributions of information-theoretic measures between A1 L4 and A1 L2/3 were compared using Kruskal-Wallis tests with post-hoc pairwise tests. We chose the $100 \mathrm{~ms}$ window size (50 ms slide) for all comparisons shown in the main manuscript. Similar results were obtained across most tested window sizes (see Supplementary Information).

\section{References}

[1] Lewicki MS. Efficient coding of natural sounds. Nat Neurosci 2002; 5:356 - 63. DOI: 10.1038/nn831.

[2] Smith E, Lewicki MS. Efficient coding of time-relative structure using spikes. Neural Comput 2005; 17: 19 - 45. DOI: 10.1162/0899766052530839.

930 [3] Bauer EE, Klug A, Pollak GD. Spectral determination of responses to species-specific calls in the dorsal nucleus of the lateral lemniscus. J Neurophysiol 2002; 88: 1955-1967.

[4] Pollak GD. The dominant role of inhibition in creating response selectivities for communication calls in the brainstem auditory system. Hear Res 2013; 305: 86 - 101. DOI: 10.1016/j.heares.2013.03.001.

935 [5] Roberts PD, Portfors CV. Responses to social vocalizations in the dorsal cochlear nucleus of mice. Front Syst Neurosci 2015; 9: 1-13.

[6] Grimsley JMS, Shanbhag SJ, Palmer AR, Wallace MN. Processing of Communication Calls in Guinea Pig Auditory Cortex. PLoS One 2012; 7: e51646. DOI: 10.1371/journal.pone.0051646. 
940 [7] Wollberg Z, Newman JD. Auditory cortex of squirrel monkey: Response patterns of single cells to species-specific vocalizations. Science 1972; 212 - 4 . DOI: 10.1126/science.175.4018.212.

[8] Rauschecker JP, Tian B, Hauser M. Processing of complex sounds in the macaque nonprimary auditory cortex. Science 1995; 268: 111 - 4. DOI: 10.1126/science.7701330.

945 [9] Tian B, Reser D, Durham A, Kustov A, Rauschecker JP. Functional specialization in rhesus monkey auditory cortex. Science 2001; 292: 290-293.

[10] Petkov Cl, Kayser C, Steudel T,Whittingstall K, Augath M, Logothetis NK. A voice region in the monkey brain. Nat Neurosci 2008; 11: 367-374.

[11] Perrodin C, Kayser C, Logothetis NK, Petkov Cl. Voice cells in the primate temporal lobe. Curr Biol 2011; 21: 1408-1415.

[12] Sadagopan S, Temiz-Karayol NZ, Voss HU. High-field functional magnetic resonance imaging of vocalization processing in marmosets. Sci Rep 2015; 5: 10950.

[13] Atencio CA, Sharpee TO, Schreiner CE. Receptive field dimensionality increases from the auditory midbrain to cortex. J Neurophysiol 2012; 107: 2594-2603.

955 [14] Chechik G, Anderson MJ, Bar-Yosef O, Young ED, Tishby N, Nelken I. Reduction of information redundancy in the ascending auditory pathway. Neuron 2006; 51: 359-368.

[15] Šuta D, Popelář J, Kvašňák E, Syka J. Representation of species-specific vocalizations in the medial geniculate body of the guinea pig. Exp Brain Res 2007; 183: 377-388.

[16] Šuta D, Kvašňák E, Popelář J, Syka J. Representation of Species-Specific Vocalizations in the Inferior Colliculus of the Guinea Pig. J Neurophysiol 2003; 90: 3794-3808.

[17] Šuta D, Popelář J, Burianová J, Syka J. Cortical Representation of Species-Specific Vocalizations in Guinea Pig. PLoS One 2013; 8: e65432.

[18] Wang X, Merzenich MM, Beitel R, Schreiner CE. Representation of a species-specific vocalization in the primary auditory cortex of the common marmoset: Temporal and spectral characteristics. J Neurophysiol 1995; 74: 2685-2706.

[19] Wang X, Kadia SC. Differential representation of species-specific primate vocalizations in the auditory cortices of marmoset and cat. J Neurophysiol 2001; 86: 2616-2620.

[20] Glass I, Wollberg Z. Auditory Cortex Responses to Sequences of Normal and Reversed Squirrel Monkey Vocalizations. Brain Behav Evol 1983; 22:13 - 21. DOI: 10.1159/000121503.

[21] Newman JD, Wollberg Z. Multiple coding of species-specific vocalizations in the auditory cortex of squirrel monkeys. Brain Res 1973; 54: 287-304.

[22] Symmes D, Alexander GE, Newman JD. Neural processing of vocalizations and artificial 
stimulin the medial geniculate body of squirrel monkey. Hear Res 1980; 3: 133-146.

975 [23] Winter P, Funkenstein HH. The Effect of Species-Specific Vocalization on the Discharge of Auditory Cortical Cells in the Awake Squirrel Monkey (Saimiri sciureus). Exp Brain Res 1973; 18: 489 - 504. DOI: 10.1007/BF00234133.

[24] Aitkin L, Tran L, Syka J. The responses of neurons in subdivisions of the inferior colliculus of cats to tonal, noise and vocal stimuli. Exp Brain Res 1994; 98: 53-64.

980 [25] Buchwald J, Dickerson L, Harrison J, Hinman C. Medial geniculate body unit responses to cat cries. In: Auditory pathway. Springer, 1988, pp. 319-322.

[26] Komiya H, Eggermont JJ. Neuronal responses in cat primary auditory cortex to natural and altered species-specific calls. Hear Res 2000; 150: 27-42.

[27] Gourévitch B, Eggermont JJ. Spatial representation of neural responses to natural and altered conspecific vocalizations in cat auditory cortex. J Neurophysiol 2007; 97: 144-158.

[28] Agamaite JA, Chang C-J, Osmanski MS, Wang X. A quantitative acoustic analysis of the vocal repertoire of the common marmoset (Callithrix jacchus). J Acoust Soc Am 2015; 138: 2906-2928.

[29] Liu ST, Montes-Lourido P, Wang X, Sadagopan S. Optimal features for auditory categorization. Nat Commun 2019; 10: 1-14.

[30] Šuta D, Popelář J, Syka J. Coding of communication calls in the subcortical and cortical structures of the auditory system. Physiol Res 2008; 57 Suppl 3: S149 - 59.

[31] Sadagopan S, Wang $X$. Nonlinear spectrotemporal interactions underlying selectivity for complex sounds in auditory cortex. J Neurosci 2009; 29: 11192-11202.

995 [32] Gaucher Q, Huetz C, Gourévitch B, Laudanski J, Occelli F, Edeline JM. How do auditory cortex neurons represent communication sounds? Hear Res 2013; 305: 102-112.

[33] Moerel M, De Martino F, Uğurbil K, Yacoub E, Formisano E. Processing complexity increases in superficial layers of human primary auditory cortex. Sci Rep 2019; 9: 1-9.

[34] Berryman JC. Guinea-pig vocalizations: Their structure, causation and function. Zeitschrift für Tierpsychologie 1976; 41(1): 80-106.

[35] Eisenberg JF (1974), In: Weir BJ, Rowlands IW. Biology of hystricomorph rodents; 1974. pp. 211-244.

[36] Redies H, Sieben U, Creutzfeldt OD. Functional subdivisions in the auditory cortex of the guinea pig. J Comp Neurol 1989; 282: 473-488.

1005 [37] Wallace MN, Rutkowski RG, Palmer AR. Identification and localisation of auditory areas in guinea pig cortex. Exp Brain Res 2000; 132: 445-456.

[38] Kajikawa Y, Schroeder CE. How local is the local field potential? Neuron 2011; 72 : 847 - 
58. DOI: 10.1016/j.neuron.2011.09.029.

[39] Lau B. 2-d Kolmorogov-Smirnov test, n-d energy test, Hotelling T^2 test; 2020 [cited 2020 Sept 11]. Database: GitHub [internet]. Available from: https://github.com/brian-lau/multdist

[40] Rolls ET, Tovee MJ. Sparseness of the neuronal representation of stimuli in the primate temporal visual cortex. J Neurophysiol 1995; 73(2): 713-26.

[41] Vinje WE, Gallant JL. Sparse coding and decorrelation in primary visual cortex during natural vision. Science 2000 18; 287(5456): 1273-6.

1015 [42] Thorson IL, Liénard J, David SV. The essential complexity of auditory receptive fields. PLoS Comp Biol 2015; 11(12): e1004628.

[43] Pennington JR, David SV. Complementary effects of adaptation and gain control on sound encoding in primary auditory cortex. Eneuro 2020; 7(6).

[44] Rahman M, Willmore BD, King AJ, Harper NS. Simple transformations capture auditory input to cortex. Proc Natl Acad Sci U S A 2020; 117(45): 28442-51.

[45] Feng L, Wang X. Harmonic template neurons in primate auditory cortex underlying complex sound processing. Proc Natl Acad Sci U S A 2017; 114(5): E840-8.

[46] Atiani S, David SV, Elgueda D, Locastro M, Radtke-Schuller S, Shamma SA, et al. Emergent selectivity for task-relevant stimuli in higher-order auditory cortex. Neuron. 2014; 82(2): 486-99.

[47] Elgueda D, Duque D, Radtke-Schuller S, Yin P, David SV, Shamma SA, et al. Statedependent encoding of sound and behavioral meaning in a tertiary region of the ferret auditory cortex. Nat Neurosci 2019; 22(3): 447-59.

[48] Cover TM. Elements of information theory. John Wiley \& Sons, 1999.

1030 [49] Liu RC, Schreiner CE. Auditory cortical detection and discrimination correlates with communicative significance. PLoS Biol 2007; 5: e173.

[50] Strong SP, Koberle R, Van Steveninck RRDR, Bialek W. Entropy and information in neural spike trains. Phys Rev Lett 1998; 80: 197.

[51] Vinje WE, Gallant JL. Natural stimulation of the nonclassical receptive field increases information transmission efficiency in V1. J Neurosci 2002; 22: 2904-2915.

[52] Reinagel P, Reid RC. Temporal coding of visual information in the thalamus. J Neurosci 2000; 20: 5392-5400.

[53] Souffi S, Lorenzi C, Varnet L, Huetz C, Edeline JM. Noise-Sensitive but More Precise Subcortical Representations Coexist with Robust Cortical Encoding of Natural 1040 Vocalizations. J Neurosci 2020; 40: 5228 - 5246. DOI: 10.1523/JNEUROSCI.273119.2020. 
[54] Butts DA. How much information is associated with a particular stimulus? Netw Comput Neural Syst 2003; 14: 177-187.

[55] Butts DA, Goldman MS. Tuning curves, neuronal variability, and sensory coding. PLoS Biol 2006; 4: e92.

[56] Montgomery N, Wehr M. Auditory cortical neurons convey maximal stimulus-specific information at their best frequency. J Neurosci 2010; 30: 13362-13366.

[57] Wallace MN, Rutkowski RG, Palmer AR. Responses to the purr call in three areas of the guinea pig auditory cortex. Neuroreport 2005; 16: 2001-2005.

1050 [58] Portfors C V, Roberts PD, Jonson K. Over-representation of species-specific vocalizationwas in the awake mouse inferior colliculus. Neuroscience 2009; 162: 486-500.

[59] Garcia-Lazaro JA, Shepard KN, Miranda JA, Liu RC, Lesica NA. An overrepresentation of high frequencies in the mouse inferior colliculus supports the processing of ultrasonic vocalizations. PLoS One 2015; 10(8): e0133251.

1055 [60] Klug A, Bauer EE, Hanson JT, Hurley L, Meitzen J, Pollack GD. Response selectivity for species-specific calls in the inferior colliculus of Mexican free-tailed bats is generated by inhibition. J Neurophysiol 2002; 88: 1941-1954.

[61] Tanaka H, Taniguchi I. Responses of medial geniculate neurons to species-specific vocalized sounds in the guinea pig. Jpn J Physiol 1991; 41: 817-829.

1060 [62] Philibert B, Laudanski J, Edeline JM. Auditory thalamus responses to guinea-pig vocalizations: A comparison between rat and guinea-pig. Hear Res 2005; 209: 97-103.

[63] Hromádka T, DeWeese MR, Zador AM. Sparse representation of sounds in the unanesthetized auditory cortex. PLoS Biol 2008; 6: e16.

[64] Atencio CA, Schreiner CE. Laminar diversity of dynamic sound processing in cat primary auditory cortex. J Neurophysiol 2010; 103: 192-205.

[65] Atencio CA, Sharpee TO, Schreiner CE. Hierarchical computation in the canonical auditory cortical circuit. Proc Natl Acad Sci U S A 2009; 106: 21894-21899.

[66] Sharpee TO, Atencio CA, Schreiner CE. Hierarchical representations in the auditory cortex. Curr Opin Neurobiol 2011; 21: 761 - 7. DOI: 10.1016/j.conb.2011.05.027.

1070 [67] Branco T, Clark BA, Häusser M. Dendritic discrimination of temporal input sequences in cortical neurons. Science 2010; 329(5999): 1671-5.

[68] Kerlin A, Mohar B, Flickinger D, MacLennan BJ, Dean MB, Davis C, Spruston N, Svoboda K. Functional clustering of dendritic activity during decision-making. Elife 2019; 8: e46966.

[69] Hemberger M, Shein-Idelson M, Pammer L, Laurent G. Reliable sequential activation of 1075 neural assemblies by single pyramidal cells in a three-layered cortex. Neuron. 2019; 
104(2): 353-69.

[70] Riesenhuber M, Poggio T. Hierarchical models of object recognition in cortex. Nat Neurosci 1999; 2: 1019-1025.

[71] Liu BH, Wu GK, Arbuckle R, Tao HW, Zhang LI. Defining cortical frequency tuning with

1080 recurrent excitatory circuitry. Nat Neurosci 2007; 10: 1594 - 600. DOI: 10.1038/nn2012.

[72] Wu GK, Arbuckle R, Liu B, Tao HW, Zhang LI. Lateral sharpening of cortical frequency tuning by approximately balanced inhibition. Neuron 2008; 58: 132-143.

[73] Sadagopan S, Wang X. Contribution of inhibition to stimulus selectivity in primary auditory cortex of awake primates. J Neurosci 2010; 30: 7314-7325.

1085 [74] Gaucher Q, Huetz C, Gourévitch B, Edeline JM. Cortical inhibition reduces information redundancy at presentation of communication sounds in the primary auditory cortex. $J$ Neurosci 2013; 33: 10713-10728.

[75] Gaucher Q, Yger P, Edeline JM. Increasing excitation versus decreasing inhibition in auditory cortex: consequences on the discrimination performance between communication sounds. J Physiol 2020; 598: 3765 - 3785. DOI: 10.1113/JP279902.

[76] Kato HK, Asinof SK, Isaacson JS. Network-Level Control of Frequency Tuning in Auditory Cortex. Neuron 2017; 95: 412 - 423. DOI: 10.1016/j.neuron.2017.06.019.

[77] Olshausen BA, Field DJ. Sparse coding of sensory inputs. Curr Opin Neurobiol 2004; 14 : 481-487.

1095 [78] DiCarlo JJ, Cox DD. Untangling invariant object recognition. Trends Cogn Sci 2007; 11: 333-341.

[79] Sadagopan S, Wang X. Level invariant representation of sounds by populations of neurons in primary auditory cortex. $J$ Neurosci 2008; 28: 3415-3426.

[80] Tchernichovski O, Nottebohm F, Ho CE, Pesaran B, Mitra PP. A procedure for an automated measurement of song similarity. Anim Behav 2000; 59: 1167-1176.

[81] Luparello TJ. Stereotaxic atlas of the forebrain of the guinea pig. Karger Basel, 1967.

[82] Redies $\mathrm{H}$, Brandner S, Creutzfeldt OD. Anatomy of the auditory thalamocortical system of the guinea pig. J Comp Neurol 1989; 282: 489 - 511. DOI: 10.1002/cne.902820403.

[83] Anderson LA, Wallace MN, Palmer AR. Identification of subdivisions in the medial geniculate body of the guinea pig. Hear Res 2007; 228: 156-167.

[84] Wallace MN, Anderson LA, Palmer AR. Phase-locked responses to pure tones in the auditory thalamus. J Neurophysiol 2007; 98: 1941 - 52. DOI: 10.1152/jn.00697.2007.

[85] Issa EB, Wang X. Sensory responses during sleep in primate primary and secondary auditory cortex. J Neurosci 2008; 28: 14467-14480. 
1110 [86] Hanes DP, Thompson KG, Schall JD. Relationship of presaccadic activity in frontal eye field and supplementary eye field to saccade initiation in macaque: Poisson spike train analysis. Exp Brain Res 1995; 103: 85 - 96. DOI: 10.1007/BF00241967.

[87] Legendy CR, Salcman M. Bursts and recurrences of bursts in the spike trains of spontaneously active striate cortex neurons. J Neurophysiol 1985; 53: 926-939.

1115 [88] Sheinberg DL, Logothetis NK. Noticing familiar objects in real world scenes: the role of temporal cortical neurons in natural vision. J Neurosci 2001; 21: 1340-1350.

[89] Lehky SR, Sejnowski TJ, Desimone R. Selectivity and sparseness in the responses of striate complex cells. Vision Res 2005; 45: 57-73.

\section{Acknowledgements}

We thank Dr. Yi Zhou (ASU) for insightful comments on the manuscript. We thank Isha Kumbam and Samuel Li for recording and classifying guinea pig vocalizations, Dr. Marianny Pernia, Shi Tong Liu, and Dr. Flora Antunes for assistance with electrophysiological experiments, and Dr. 1125 Marianny Pernia for assistance with analysis. We thank Stacy Cashman and Mark Petts for surgical support; Dr. Amanda Fisher for veterinary support; and Jillian Harr, Sarah Gray, Julia Skrinjar, Brent Barbe, and Elizabeth Chasky for animal care. SS is grateful for support from the NIH (R01DC017141), a Pennsylvania Lions Hearing Research Foundation grant, and a NARSAD Young Investigator grant from the Brain and Behavior Foundation. 